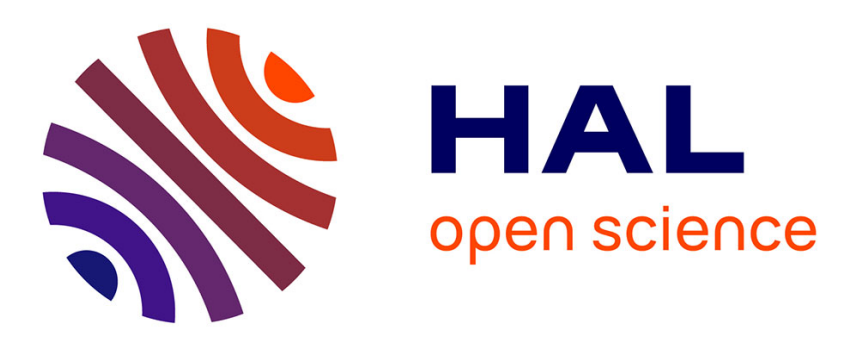

\title{
An implicit hybridized discontinuous Galerkin method for the 3D time-domain Maxwell equations
}

\author{
Alexandra Christophe, Stéphane Descombes, Stéphane Lanteri
}

\section{To cite this version:}

Alexandra Christophe, Stéphane Descombes, Stéphane Lanteri. An implicit hybridized discontinuous Galerkin method for the 3D time-domain Maxwell equations. Applied Mathematics and Computation, 2018, 319, pp.395 - 408. 10.1016/j.amc.2017.04.023 . hal-01674044

\section{HAL Id: hal-01674044 \\ https://hal.inria.fr/hal-01674044}

Submitted on 8 Feb 2018

HAL is a multi-disciplinary open access archive for the deposit and dissemination of scientific research documents, whether they are published or not. The documents may come from teaching and research institutions in France or abroad, or from public or private research centers.
L'archive ouverte pluridisciplinaire $\mathbf{H A L}$, est destinée au dépôt et à la diffusion de documents scientifiques de niveau recherche, publiés ou non, émanant des établissements d'enseignement et de recherche français ou étrangers, des laboratoires publics ou privés. 


\title{
An implicit hybridized discontinuous Galerkin method for the 3D time-domain Maxwell equations
}

\author{
Alexandra Christophe-Argenvillier ${ }^{\mathrm{b}}$, Stéphane Descombes ${ }^{\mathrm{a}, *}$, Stéphane Lanteri $^{\mathrm{b}}$ \\ ${ }^{a}$ Université Côte d'Azur, CNRS, Inria, LJAD, France, \\ ${ }^{b}$ Université Côte d'Azur, Inria, CNRS, LJAD, France
}

\begin{abstract}
We present a time-implicit hybridizable discontinuous Galerkin (HDG) method for numerically solving the system of three-dimensional (3D) time-domain Maxwell equations. This method can be seen as a fully implicit variant of classical so-called DGTD (Discontinuous Galerkin Time-Domain) methods that have been extensively studied during the last 10 years for the simulation of time-domain electromagnetic wave propagation. The proposed method has been implemented for dealing with general 3D problems discretized using unstructured tetrahedral meshes. We provide numerical results aiming at assessing its numerical convergence properties by considering a model problem on one hand, and its performance when applied to more realistic problems. We also include some performance comparisons with a centered flux time-implicit DGTD method.
\end{abstract}

Keywords: Maxwell's equations, time-domain, implicit time stepping, discontinuous Galerkin, hybridized discontinuous Galerkin

\section{Introduction}

\subsection{Generalities about the DGTD method}

During the last ten years, the DGTD method has progressively emerged as a viable alternative to well established FDTD (Finite Difference Time-Domain) [1] and FETD (Finite Element Time-Domain) [2] methods for the numerical simulation of electromagnetic wave propagation problems in the time-domain.

\footnotetext{
${ }^{*}$ Corresponding author.

Email addresses: alexandra.christophe-argenvillier@inria.fr (Alexandra Christophe-Argenvillier), stephane.descombes@unice.fr (Stéphane Descombes ), stephane.lanteri@inria.fr (Stéphane Lanteri)
} 
The DGTD method can be considered as a finite element method where the continuity constraint at an element interface is released. While it keeps almost all the advantages of the finite element method (large spectrum of applications, complex geometries, etc.), the DGTD method has other nice properties which explain the renewed interest it gains in various domains in scientific computing:

- It is naturally adapted to a high order approximation of the unknown field. Moreover, one may increase the degree of the approximation in the whole mesh as easily as for spectral methods but, with a DGTD method, this can also be done locally i.e. at the mesh cell level. In most cases, the approximation relies on a polynomial interpolation method but the method also offers the flexibility of applying local approximation strategies that best fit to the intrinsic features of the modeled physical phenomena.

- When the discretization in space is coupled to an explicit time integration method, the DG method leads to a block diagonal mass matrix independently of the form of the local approximation (e.g the type of polynomial interpolation). This is a striking difference with classical, continuous FETD formulations. Moreover, the mass matrix is diagonal if an orthogonal basis is chosen.

- It easily handles complex meshes. The grid may be a classical conforming finite element mesh, a non-conforming one or even a hybrid mesh made of various elements (tetrahedra, prisms, hexahedra, etc.). The DGTD method has been proven to work well with highly locally refined meshes. This property makes the DGTD method more suitable to the design of a $h p$-adaptive solution strategy (i.e. where the characteristic mesh size $h$ and the interpolation degree $p$ changes locally wherever it is needed).

- It is flexible with regards to the choice of the time stepping scheme. One may combine the discontinuous Galerkin spatial discretization with any global or local explicit time integration scheme, or even implicit, provided the resulting scheme is stable.

- It is naturally adapted to parallel computing. As long as an explicit time integration scheme is used, the DGTD method is easily parallelized. Moreover, the compact nature of method is in favor of high computation to communication ratio especially when the interpolation order is increased.

As in a classical finite element framework, a discontinuous Galerkin formulation relies on a weak form of the continuous problem at hand. However, due to the discontinuity of the global approximation, this variational formulation has to be defined at the 
element level. Then, a degree of freedom in the design of a discontinuous Galerkin scheme stems from the approximation of the boundary integral term resulting from the application of an integration by parts to the element-wise variational form. In the spirit of finite volume methods, the approximation of this boundary integral term calls for a numerical flux function which can be based on either a centered scheme or an upwind scheme, or a blend of these two schemes.

\subsection{DGTD methods for time-domain electromagnetics}

In the early 2000's, DGTD methods for time-domain electromagnetics have been studied by a few groups of researchers, most of then from the applied mathematics community. One of the most significant contributions is due to Hesthaven and Warburton [3] in the form of a high order nodal DGTD method formulated on unstructured simplicial meshes. The proposed formulation is based on an upwind numerical flux, nodal basis expansions on a triangle (2D case) and a tetrahedron (3D case) and a Runge-Kutta time stepping scheme. In [4], Kakbian et al. describe a rather similar approach. More precisely, the authors develop a parallel, unstructured, high order DGTD method based on simple monomial polynomials for spatial discretization, an upwind numerical flux and a fourth-order Runge-Kutta scheme for time marching. The method has been implemented with hexahedral and tetrahedral meshes. A high order DGTD method based on a strong stability preserving Runge-Kutta time scheme has been studied by Chen et al. [5]. The authors also present post-processing techniques that can double the convergence order. A locally divergence-free DGTD method is formulated and studied by Cockburn et al. in [6]. In the same period, a high order nodal DGTD method formulated on unstructured simplicial meshes has also been proposed by Fezoui et al. [7]. However, contrary to the DGTD methods discussed in [3] and [4], the DGTD method in [7] is non-dissipative thanks to a combination of a centered numerical flux with a second-order leap-frog time stepping scheme. The DGTD method has then been progressively considered and extended to increasinlgy more complex modeling situations by groups of researchers in the applied electromagnetics and electrical engineering communities for a wide variety of applications related to aeronautics, defense, semiconductor device fabrication, etc. [8]-[9]-[10]-[11]-[12]-[13]-[14] to cite a few. More recently, the method has also been adopted and further developed by researchers in the nano-optics domain [15]-[16][17]-[18]. A full review of the nowadays numerous applications of DGDT methods would certainly require than a simple paragraph. Also worth to note, the DGTD method has been implement in commercial software such HFSS-TD (the time-domain version of the well-known HFSS software used for antenna design) [19]. 


\subsection{Explicit versus implicit DGTD methods}

From the above discussion, it is clear that the DGTD method is nowadays a very popular numerical method in the computational electromagnetics community. The works mentioned so far are mostly concerned with time explicit DGTD methods relying on the use of a single global time step computed so as to ensure stability of the simulation. It is however well known that when combined with an explicit time integration method and in the presence of an unstructured locally refine mesh, a high order DGTD method suffers from a severe time step size restriction. A possible alternative to overcome this limitation is to use smaller time steps, given by a local stability criterion, precisely where the smallest elements are located. The local character of a DG formulation is a very attractive feature for the development of explicit local time stepping schemes [20]-[21]-[22]. An alternative approach that has been considered in [23]-[24] is to use a hybrid explicit-implicit (or locally implicit) time integration strategy. Such a strategy relies on a component splitting deduced from a partitioning of the mesh cells in two sets respectively gathering coarse and fine elements. In these works, a second-order explicit leap-frog scheme is combined with a second-order implicit Crank-Nicolson scheme in the framework of a non-dissipative (centered flux based) DG discretization in space. At each time step, a large linear system must be solved whose structure is partly diagonal (for those rows of the system associated to the explicit unknowns) and partly sparse (for those rows of the system associated to the implicit unknowns). The computational efficiency of this locally implicit DGTD method depends on the size of the set of fine elements that directly inluences the size of the sparse part of the matrix system. Therefore, an approach for reducing the size of the subsystem of globally coupled (i.e. implicit) unknowns is worth considering if one wants to solver very large-scale problems.

A particularly appealing solution in this context is given by the concept of hybridizable discontinuous Galerkin (HDG) method. The HDG method has been first introduced by Cockbrun et al. in [25] for a model elliptic probelm and has been subsequently developed for a variety of PDE systems in continuum mechanics [26]. The essential ingredients of a HDG method are a local Galerkin projection of the underlying system of PDEs at the element level onto spaces of polynomials to parametrize the numerical solution in terms of the numerical trace; a judicious choice of the numerical flux to provide stability and consistency; and a global jump condition that enforces the continuity of the numerical flux to arrive at a global weak formulation in terms of the numerical trace. The HDG methods are fully implicit, high-order accurate and endowed with several unique features which distinguish themselves from

other discontinuous Galerkin methods. mots importantly, they reduce the globally coupled unknowns to the approximate trace of the solution on element boundaries, 
thereby leading to a significant reduction in the degrees of freedom. HDG methods for the system of time-harmonic Maxwell equations have been introduced in [27]-[28]-[29].

\subsection{Objectives of this work}

In view of devising a hybrid explicit-implicit HDG method, a preliminary step which is the focus of this work is to develop a fully implicit HDG formulation. In this paper we present such a time-implicit hybridizable discontinuous Galerkin (HDG) method for numerically solving the system of three-dimensional (3D) time-domain Maxwell equations. The method is based on a second-order Crank-Nicolson scheme for time integration whereas the discretization in space and in particular the definition of HDG traces are mostly inspired from the HDG formulations already knonw for the time-harmonic Maxwell equations [27]-[28]-[29]. We study the stability of this time-implicit HDGTD (Hybridizable Discontinuous Galerkin Time-Domain) method as well as the solvability of the reduced system for the hybrid variable. The sequel of the paper is oragnized as follows: in section 2 we state the initial and boundary value problem that we want to solve, and then introduce some notations for the sequel; section 3 is the core of the paper where we describe and study the properties of the time-implicit HDGTD method; in section 4 we discuss about some implementation aspects of the method; preliminary results are presented in section 5; finally, section 6 concludes the paper. We note that the numerical results presented here before all aim at validating the proposed HDGTD method. A detailed assessment of the performances of the method for more realistic 3D problems simulated on parallel computing systems will be the subject of a subsequent paper.

\section{Problem statement and notations}

\subsection{The system of $3 D$ Maxwell equations}

We consider the system of 3D time domain Maxwell's equations on a bounded polyhedral domain $\Omega \subset \mathbb{R}^{3}$

$$
\left\{\begin{array}{l}
\varepsilon \partial_{t} \mathbf{E}-\operatorname{curl} \mathbf{H}=0, \text { dans } \Omega \times[0, T], \\
\mu \partial_{t} \mathbf{H}+\operatorname{curl} \mathbf{E}=0, \text { dans } \Omega \times[0, T],
\end{array}\right.
$$

where the symbol $\partial_{t}$ denotes a time derivate, $T$ a final time, $\mathbf{E}(\mathbf{x}, t)$ and $\mathbf{H}(\mathbf{x}, t)$ are the electric and magnetic fields. The dielectric permittivity tensor $\varepsilon$ and the magnetic permeability tensor $\mu$ are varying in space, time-invariant and both positive 
functions. The boundary of $\Omega$ can be decomposed in $\partial \Omega=\Gamma_{m} \cup \Gamma_{a}$ with $\Gamma_{m} \cap \Gamma_{a}=\emptyset$ and the boundary conditions are choosen as

$$
\left\{\begin{aligned}
\mathbf{n} \times \mathbf{E} & =0, \text { sur } \Gamma_{m} \times[0, T], \\
\mathbf{n} \times \mathbf{E}+\mathbf{n} \times(\mathbf{n} \times \mathbf{H}) & =\mathbf{n} \times \mathbf{E}^{\text {inc }}+\mathbf{n} \times\left(\mathbf{n} \times \mathbf{H}^{\text {inc }}\right)=\mathbf{g}^{\text {inc }}, \text { sur } \Gamma_{a} \times[0, T] .
\end{aligned}\right.
$$

Here $\mathbf{n}$ denotes the unit outward normal to $\partial \Omega$ and $\left(\mathbf{E}^{\text {inc }}, \mathbf{H}^{\text {inc }}\right)$ the incident field. The first boundary condition indicates a metallic boundary condition (referring to a perfectly conducting surface), the second relation is called absorbing and takes here the form of the Silver-Müller condition. Finally, the system is supplemented with inital conditions: $\mathbf{E}_{0}(\mathbf{x})=\mathbf{E}(\mathbf{x}, 0)$ and $\mathbf{H}_{0}(\mathbf{x})=\mathbf{H}(\mathbf{x}, 0)$.

\subsection{Notations and approximation spaces}

We consider a partition $\mathcal{T}_{h}$ of $\Omega$ into a set of tetraedra. Each non-empty intersection of two elements $K_{e}$ and $K_{g}$, with $e, g=\left\{1, . .,\left|\mathcal{T}_{h}\right|\right\}$, is called an interface. We denote by $\mathcal{F}_{h}^{I}$ the union of all interior interfaces of $\mathcal{T}_{h}$, by $\mathcal{F}_{h}^{B}$ the union of all boundary interfaces of $\mathcal{T}_{h}$, and $\mathcal{F}_{h}=\mathcal{F}_{h}^{I} \cup \mathcal{F}_{h}^{B}$. Note that $\partial \mathcal{T}_{h}$ represents all the interfaces $\partial K$ for all $K \in \mathcal{T}_{h}$. As a result, an interior interface shared by two elements appears twice in $\partial \mathcal{T}_{h}$, unlike in $\mathcal{F}_{h}$ where this interface is evaluated once.

For an interface $F \in \mathcal{F}_{h}^{I}, F=\bar{K}_{e} \cap \bar{K}_{g}$, let $\mathbf{v}^{ \pm}$be the traces of $\mathbf{v}$ on $F$ from the interior of $K_{e, g}$. On this interior face, we define mean values $\{\cdot\}$ and jumps $\llbracket \cdot \rrbracket$ as

$$
\left\{\begin{array}{l}
\{\mathbf{v}\}_{F}=\frac{1}{2}\left(\mathbf{v}^{+}+\mathbf{v}^{-}\right) \\
\llbracket \mathbf{v} \rrbracket_{F}=\mathbf{n}^{+} \times \mathbf{v}^{+}+\mathbf{n}^{-} \times \mathbf{v}^{-}
\end{array}\right.
$$

where the unit outward normal vector to $K$ is denoted by $\mathbf{n}^{ \pm}$. For the boundary faces these expressions are modified as

$$
\left\{\begin{array}{l}
\{\mathbf{v}\}_{F}=\mathbf{v}^{+} \\
\llbracket \mathbf{v} \rrbracket_{F}=\mathbf{n}^{+} \times \mathbf{v}^{+}
\end{array}\right.
$$

since we assume $\mathbf{v}$ is single-valued on the boundaries. In the following, we introduce the discontinuous finite element spaces and some basic operations on these spaces for later use. Let $\mathbb{P}_{p_{e}}\left(K_{e}\right)$ denote the space of polynomial functions of degree at most $p_{e}$ on the element $K_{e}$. The discontinuous finite element space is introduced as

$$
\mathbf{V}_{h}=\left\{\mathbf{v} \in\left[L^{2}(\Omega)\right]^{3} \mid \mathbf{v}_{\left.\right|_{K_{e}}} \in\left[\mathbb{P}_{p_{e}}\left(K_{e}\right)\right]^{3}, \quad \forall K_{e} \in \mathcal{T}_{h}\right\}
$$


where $L^{2}(\Omega)$ is the space of square integrable functions on the domain $\Omega$. The functions in $\mathbf{V}_{h}$ are continuous inside each element but be discontinuous across the interfaces between elements. In addition, we introduce a traced finite element space $\mathbf{M}_{h}$ given by

$$
\mathbf{M}_{h}=\left\{\boldsymbol{\eta} \in\left[L^{2}\left(\mathcal{F}_{h}\right)\right]^{3}\left|\boldsymbol{\eta}_{\left.\right|_{F_{f}}} \in\left[\mathbb{P}_{p_{f}}\left(F_{f}\right)\right]^{3},(\boldsymbol{\eta} \cdot \mathbf{n})\right|_{F_{f}}=0, \quad \forall F_{f} \in \mathcal{F}_{h}\right\} .
$$

For two vectorial functions $\mathbf{u}$ and $\mathbf{v}$ in $\left[L^{2}(D)\right]^{3}$, we denote $(\mathbf{u}, \mathbf{v})_{D}=\int_{D} \mathbf{u} \cdot \mathbf{v} \mathrm{d} \mathbf{x}$ provided $D$ is a domain in $\mathbb{R}^{3}$, and we denote $\left\langle\mathbf{u}, \mathbf{v}>_{F}=\int_{F} \mathbf{u} \cdot \mathbf{v}\right.$ ds if $F$ is a two-dimensional face. Accordingly, for the mesh $\mathcal{T}_{h}$ we have

$$
\begin{aligned}
(\cdot, \cdot)_{\mathcal{T}_{h}} & =\sum_{K \in \mathcal{T}_{h}}(\cdot, \cdot)_{K}, & \langle\cdot, \cdot\rangle_{\partial \mathcal{T}_{h}} & =\sum_{K \in \mathcal{T}_{h}}\langle\cdot, \cdot\rangle_{\partial K}, \\
\langle\cdot, \cdot\rangle_{\mathcal{F}_{h}} & =\sum_{F \in \mathcal{F}_{h}}\langle\cdot, \cdot\rangle_{F}, & \langle\cdot, \cdot\rangle_{\Gamma_{a}} & =\sum_{F \in \mathcal{F}_{h} \cap \Gamma_{a}}\langle\cdot, \cdot\rangle_{F} \cdot
\end{aligned}
$$

We set $\mathbf{v}^{t}=-\mathbf{n} \times(\mathbf{n} \times \mathbf{v}), \quad \mathbf{v}^{n}=\mathbf{n}(\mathbf{n} \cdot \mathbf{v})$ where $\mathbf{v}^{t}$ and $\mathbf{v}^{n}$ are the tangential and normal components of $\mathbf{v}$ such as $\mathbf{v}=\mathbf{v}^{t}+\mathbf{v}^{n}$.

\section{Principles and formulation of the global HDG problem}

Following the classical DG approach, approximate solutions $\left(\mathbf{E}_{h}, \mathbf{H}_{h}\right)$, for all $t \in$ $[0, T]$, are seeked in the space $\mathbf{V}_{h} \times \mathbf{V}_{h}$ satisfying for all $K$ in $\mathcal{T}_{h}$

$$
\left\{\begin{array}{l}
\left(\varepsilon \partial_{t} \mathbf{E}_{h}, \mathbf{v}\right)_{K}-\left(\operatorname{curl} \mathbf{H}_{h}, \mathbf{v}\right)_{K}=0, \forall \mathbf{v} \in \mathbf{V}_{h} \\
\left(\mu \partial_{t} \mathbf{H}_{h}, \mathbf{v}\right)_{K}+\left(\operatorname{curl} \mathbf{E}_{h}, \mathbf{v}\right)_{K}=0, \forall \mathbf{v} \in \mathbf{V}_{h}
\end{array}\right.
$$

Applying Green's formula, on both equations of (3) introduces boundary terms which are replaced by numerical traces $\widehat{\mathbf{E}}_{h}$ and $\widehat{\mathbf{H}}_{h}$ in order to ensure the connection between element-wise solutions and global consistency of the discretization. This leads to the formulation for all $t \in[0, T]$

$$
\left\{\begin{array}{l}
\left(\varepsilon \partial_{t} \mathbf{E}_{h}, \mathbf{v}\right)_{K}-\left(\mathbf{H}_{h}, \mathbf{c u r l} \mathbf{v}\right)_{K}+\left\langle\widehat{\mathbf{H}}_{h}, \mathbf{n} \times \mathbf{v}\right\rangle_{\partial K}=0, \forall \mathbf{v} \in \mathbf{V}_{h} \\
\left(\mu \partial_{t} \mathbf{H}_{h}, \mathbf{v}\right)_{K}+\left(\mathbf{E}_{h}, \mathbf{c u r l} \mathbf{v}\right)_{K}-\left\langle\widehat{\mathbf{E}}_{h}, \mathbf{n} \times \mathbf{v}\right\rangle_{\partial K}=0, \forall \mathbf{v} \in \mathbf{V}_{h}
\end{array}\right.
$$

It is straightforward to verify that $\mathbf{n} \times \mathbf{v}=\mathbf{n} \times \mathbf{v}^{t}$ and $\langle\mathbf{H}, \mathbf{n} \times \mathbf{v}\rangle=-\langle\mathbf{n} \times \mathbf{H}, \mathbf{v}\rangle$. Therefore, using numerical traces defined in terms of the tangential components $\widehat{\mathbf{H}}_{h}^{t}$ 
and $\widehat{\mathbf{E}}_{h}^{t}$, we can rewrite (4) as

$$
\left\{\begin{array}{l}
\left(\varepsilon \partial_{t} \mathbf{E}_{h}, \mathbf{v}\right)_{K}-\left(\mathbf{H}_{h}, \mathbf{c u r l} \mathbf{v}\right)_{K}+\left\langle\widehat{\mathbf{H}}_{h}^{t}, \mathbf{n} \times \mathbf{v}\right\rangle_{\partial K}=0, \forall \mathbf{v} \in \mathbf{V}_{h}, \\
\left(\mu \partial_{t} \mathbf{H}_{h}, \mathbf{v}\right)_{K}+\left(\mathbf{E}_{h}, \mathbf{c u r l} \mathbf{v}\right)_{K}-\left\langle\widehat{\mathbf{E}}_{h}^{t}, \mathbf{n} \times \mathbf{v}\right\rangle_{\partial K}=0, \forall \mathbf{v} \in \mathbf{V}_{h} .
\end{array}\right.
$$

The hybrid variable $\boldsymbol{\Lambda}_{h}$ introduced in the setting of a HDG method is here defined for all the interfaces of $\mathcal{F}_{h}$ as

$$
\Lambda_{h}:=\widehat{\mathbf{H}}_{h}^{t}, \quad \forall F \in \mathcal{F}_{h} .
$$

We want to determine the fields $\widehat{\mathbf{H}}_{h}^{t}$ and $\widehat{\mathbf{E}}_{h}^{t}$ in each element $K$ of $\mathcal{T}_{h}$ by solving system (5) and assuming that $\boldsymbol{\Lambda}_{h}$ is known on all the faces of an element $K$. Let us consider a numerical trace $\widehat{\mathbf{E}}_{h}^{t}$ for all $K$ given

$$
\widehat{\mathbf{E}}_{h}^{t}=\mathbf{E}_{h}^{t}+\tau_{K} \mathbf{n} \times\left(\boldsymbol{\Lambda}_{h}-\mathbf{H}_{h}^{t}\right) \text { on } \partial K,
$$

where $\tau_{K}$ is a local stabilization parameter.

Remark 1. In a classical DG method the traces of the local fields $\mathbf{E}_{h}$ and $\mathbf{H}_{h}$ between neighboring elements are defined as

$$
\widehat{\mathbf{E}}_{h}=\left\{\mathbf{E}_{h}\right\}+\alpha_{H} \llbracket \mathbf{H}_{h} \rrbracket \text { and } \widehat{\mathbf{H}}_{h}=\left\{\mathbf{H}_{h}\right\}+\alpha_{E} \llbracket \mathbf{E}_{h} \rrbracket,
$$

where $\alpha_{H}$ and $\alpha_{E}$ are positive penalty parameters.

Remark 2. Following the HDG approach, when the hybrid variable $\boldsymbol{\Lambda}_{h}$ is known for all the faces of the element $K$, the electromagnetic field into $K_{e}$ can be determined by the local system (5) using (6) and (7).

Adding the contributions of (5) over all the elements and enforcing the continuity of the tangential component of $\widehat{\mathbf{E}}_{h}$, we can formulate a problem which is to find $\left(\mathbf{E}_{h}, \mathbf{H}_{h}, \boldsymbol{\Lambda}_{h}\right) \in \mathbf{V}_{h} \times \mathbf{V}_{h} \times \mathbf{M}_{h}$ such that for all $t \in[0, T]$

$$
\begin{aligned}
& \left(\varepsilon \partial_{t} \mathbf{E}_{h}, \mathbf{v}\right)_{\mathcal{T}_{h}}-\left(\mathbf{H}_{h}, \mathbf{c u r l} \mathbf{v}\right)_{\mathcal{T}_{h}}+\left\langle\boldsymbol{\Lambda}_{h}, \mathbf{n} \times \mathbf{v}\right\rangle_{\partial \mathcal{T}_{h}}=0, \forall \mathbf{v} \in \mathbf{V}_{h}, \\
& \left(\mu \partial_{t} \mathbf{H}_{h}, \mathbf{v}\right)_{\mathcal{T}_{h}}+\left(\mathbf{E}_{h}, \mathbf{c u r l} \mathbf{v}\right)_{\mathcal{T}_{h}}-\left\langle\widehat{\mathbf{E}}_{h}^{t}, \mathbf{n} \times \mathbf{v}\right\rangle_{\partial \mathcal{T}_{h}}=0, \forall \mathbf{v} \in \mathbf{V}_{h}, \\
& \left\langle\llbracket \widehat{\mathbf{E}}_{h} \rrbracket, \boldsymbol{\eta}\right\rangle_{\mathcal{F}_{h}}-\left\langle\boldsymbol{\Lambda}_{h}, \boldsymbol{\eta}\right\rangle_{\Gamma_{a}}=\left\langle\mathbf{g}^{\text {inc }}, \boldsymbol{\eta}\right\rangle_{\Gamma_{a}}, \forall \boldsymbol{\eta} \in \mathbf{M}_{h},
\end{aligned}
$$

where the last equation is called the conservativity condition with which we ask the tangential component of $\widehat{\mathbf{E}}_{h}$ to be weakly continuous across any interface between 
two neighboring elements. With the definition (7), we can apply a Green formula on the second equation of (8) and get for all $t \in[0, T]$

$$
\begin{aligned}
& \left(\varepsilon \partial_{t} \mathbf{E}_{h}, \mathbf{v}\right)_{\mathcal{T}_{h}}-\left(\mathbf{H}_{h}, \mathbf{c u r l} \mathbf{v}\right)_{\mathcal{T}_{h}}+\left\langle\boldsymbol{\Lambda}_{h}, \mathbf{n} \times \mathbf{v}\right\rangle_{\partial \mathcal{T}_{h}}=0, \forall \mathbf{v} \in \mathbf{V}_{h}, \\
& \left(\mu \partial_{t} \mathbf{H}_{h}, \mathbf{v}\right)_{\mathcal{T}_{h}}+\left(\mathbf{c u r l} \mathbf{E}_{h}, \mathbf{v}\right)_{\mathcal{T}_{h}}+\left\langle\tau \mathbf{n} \times\left(\mathbf{H}_{h}-\boldsymbol{\Lambda}_{h}\right), \mathbf{n} \times \mathbf{v}\right\rangle_{\partial \mathcal{T}_{h}}=0, \forall \mathbf{v} \in \mathbf{V}_{h}, \\
& \left\langle\mathbf{n} \times \mathbf{E}_{h}, \boldsymbol{\eta}\right\rangle_{\partial \mathcal{T}_{h}}+\left\langle\tau\left(\mathbf{H}_{h}^{t}-\boldsymbol{\Lambda}_{h}\right), \boldsymbol{\eta}\right\rangle_{\partial \mathcal{T}_{h}}-\left\langle\boldsymbol{\Lambda}_{h}, \boldsymbol{\eta}\right\rangle_{\Gamma_{a}}=\left\langle\mathbf{g}^{\mathrm{inc}}, \boldsymbol{\eta}\right\rangle_{\Gamma_{a}}, \forall \boldsymbol{\eta} \in \mathbf{M}_{h} .
\end{aligned}
$$

The main principles of the HDG method can be summarized as:

1. The DoFs of the hybrid variable are determined by solving a global linear system (from the discretization of the conservation condition) supported by the interfaces of $\mathcal{F}_{h}$;

2. The DoFs of the electromagnetic field in each element are evaluated by solving local linear systems, more exactly for the DoFs of $\left(\mathbf{E}_{h}, \mathbf{H}_{h}\right)$ in the considered element.

\subsection{Stability and conservation properties when $\Gamma_{a}=\emptyset$}

Selecting $\mathbf{v}=\mathbf{E}_{h}(t)$ in the first relation of (9) and $\mathbf{v}=\mathbf{H}_{h}(t)$ in the second relation of (9) and summing the two equations we obtain

$$
\begin{aligned}
\frac{1}{2} \frac{d}{d t}\left(\varepsilon\left\|\mathbf{E}_{h}(t)\right\|^{2}+\mu\left\|\mathbf{H}_{h}(t)\right\|^{2}\right)= & -\left\langle\boldsymbol{\Lambda}_{h}, \mathbf{n} \times \mathbf{E}_{h}(t)\right\rangle_{\partial \mathcal{T}_{h}} \\
& -\left\langle\tau \mathbf{n} \times\left(\mathbf{H}_{h}(t)-\boldsymbol{\Lambda}_{h}\right), \mathbf{n} \times \mathbf{H}_{h}(t)\right\rangle_{\partial \mathcal{T}_{h}} .
\end{aligned}
$$

When $\Gamma_{a}=\emptyset$ it follows from the third relation of (8) with $\eta=\boldsymbol{\Lambda}_{h}$ that

$$
\frac{1}{2} \frac{d}{d t}\left(\varepsilon\left\|\mathbf{E}_{h}(t)\right\|^{2}+\mu\left\|\mathbf{H}_{h}(t)\right\|^{2}\right)=-\left\langle\tau \mathbf{n} \times\left(\boldsymbol{\Lambda}_{h}-\mathbf{H}_{h}(t)\right), \mathbf{n} \times\left(\boldsymbol{\Lambda}_{h}-\mathbf{H}_{h}(t)\right)\right\rangle_{\partial \mathcal{T}_{h}} \leq 0,
$$

since $\tau>0$. Thus, the energy function $\mathcal{E}_{h}(t)=\frac{1}{2}\left(\varepsilon\left\|\mathbf{E}_{h}(t)\right\|^{2}+\mu\left\|\mathbf{H}_{h}(t)\right\|^{2}\right)$ decreases in time such that $\mathcal{E}_{h}(t) \leq \mathcal{E}_{h}(0)$, for all $t>0$. This result shows the stability in $\mathrm{L}^{2}$ sense. In particular, this method is dissipative for the considered numerical trace for $\widehat{\mathbf{E}}_{h}^{t}$ in $(7)$.

\subsection{Time integration}

The system of equations (9) can be rewriten in the form of a differential algebraic equation (DAE) such as

$$
F\left(\mathbf{E}_{h}(t), \mathbf{H}_{h}(t), \boldsymbol{\Lambda}_{h}(t)\right)=0
$$


where $F$ is defined for all $\mathbf{v}$ in $\mathbf{V}_{h}$ and for all $\boldsymbol{\eta}$ in $\mathbf{M}_{h}$ by

$$
\left[\begin{array}{c}
\left(\varepsilon \partial_{t} \mathbf{E}_{h}, \mathbf{v}\right)_{\mathcal{T}_{h}}-\left(\mathbf{H}_{h}, \mathbf{c u r l} \mathbf{v}\right)_{\mathcal{T}_{h}}+\left\langle\boldsymbol{\Lambda}_{h}, \mathbf{n} \times \mathbf{v}\right\rangle_{\partial \mathcal{T}_{h}} \\
\left(\mu \partial_{t} \mathbf{H}_{h}, \mathbf{v}\right)_{\mathcal{T}_{h}}+\left(\mathbf{c u r l} \mathbf{E}_{h}, \mathbf{v}\right)_{\mathcal{T}_{h}}+\left\langle\tau \mathbf{n} \times\left(\mathbf{H}_{h}-\boldsymbol{\Lambda}_{h}\right), \mathbf{n} \times \mathbf{v}\right\rangle_{\partial \mathcal{T}_{h}} \\
\left\langle\mathbf{n} \times \mathbf{E}_{h}, \boldsymbol{\eta}\right\rangle_{\partial \mathcal{T}_{h}}+\left\langle\tau\left(\mathbf{H}_{h}^{t}-\boldsymbol{\Lambda}_{h}\right), \boldsymbol{\eta}\right\rangle_{\partial \mathcal{T}_{h}}-\left\langle\boldsymbol{\Lambda}_{h}, \boldsymbol{\eta}\right\rangle_{\Gamma_{a}}-\left\langle\mathbf{g}^{\mathrm{inc}}, \boldsymbol{\eta}\right\rangle_{\Gamma_{a}}
\end{array}\right]
$$

As explain in [30] the defined system is considered as a semi-explicit DAE. In this sense, the third equation of (10) is called algebraic equation and is considered as a constraint on the global system. The idea here is to transform the DAE into an ODE which can be straightforward solved using numerical methods. From the previews DAE, a perturbation problem is considered such as for all $t \in[0, T]$

$$
F\left(\widehat{\mathbf{E}}_{h}(t), \widehat{\mathbf{H}}_{h}(t), \widehat{\Lambda}_{h}(t)\right)=\delta\left(\widehat{\Lambda}_{h}(t)\right)
$$

with $0<\gamma<<1$ and

$$
\delta\left(\widehat{\boldsymbol{\Lambda}}_{h}(t)\right)=\left[\begin{array}{c}
0 \\
0 \\
\gamma \partial_{t} \widehat{\Lambda}_{h}
\end{array}\right]
$$

As a first step, the perturbation problem is considered

$$
\begin{gathered}
\left(\varepsilon \partial_{t} \mathbf{E}_{h}, \mathbf{v}\right)_{\mathcal{T}_{h}}-\left(\mathbf{H}_{h}, \mathbf{c u r l} \mathbf{v}\right)_{\mathcal{T}_{h}}+\left\langle\boldsymbol{\Lambda}_{h}, \mathbf{n} \times \mathbf{v}\right\rangle_{\partial \mathcal{T}_{h}}=0 \\
\left(\mu \partial_{t} \mathbf{H}_{h}, \mathbf{v}\right)_{\mathcal{T}_{h}}+\left(\mathbf{c u r l} \mathbf{E}_{h}, \mathbf{v}\right)_{\mathcal{T}_{h}}+\left\langle\tau \mathbf{n} \times\left(\mathbf{H}_{h}-\boldsymbol{\Lambda}_{h}\right), \mathbf{n} \times \mathbf{v}\right\rangle_{\partial \mathcal{T}_{h}}=0 \\
\gamma \partial_{t} \boldsymbol{\Lambda}_{h}+\left\langle\mathbf{n} \times \mathbf{E}_{h}, \boldsymbol{\eta}\right\rangle_{\partial \mathcal{T}_{h}}+\left\langle\tau\left(\mathbf{H}_{h}^{t}-\boldsymbol{\Lambda}_{h}\right), \boldsymbol{\eta}\right\rangle_{\partial \mathcal{T}_{h}}-\left\langle\boldsymbol{\Lambda}_{h}, \boldsymbol{\eta}\right\rangle_{\Gamma_{a}}=\left\langle\mathbf{g}^{\text {inc }}, \boldsymbol{\eta}\right\rangle_{\Gamma_{a}}
\end{gathered}
$$

From here we can discretize the problem in time. We consider a sequence of time steps

$$
0=t^{0}<t^{1}<t^{2}<\cdots<t^{N}=T \text {. }
$$

For $0 \leq n \leq N-1$, we denote by $\left(\mathbf{E}_{h}^{n+1}, \mathbf{H}_{h}^{n+1}, \boldsymbol{\Lambda}_{h}^{n+1}\right)$ the numerical approximations to $\left(\mathbf{E}_{h}\left(t^{n+1}\right), \mathbf{H}_{h}\left(t^{n+1}\right), \boldsymbol{\Lambda}_{h}\left(t^{n+1}\right)\right)$ at time $t^{n+1}=(n+1) \Delta t$. Using a CrankNicolson scheme on each equation of (12)-(14), the obtained system is written as: 
find $\left(\mathbf{E}_{h}^{n+1}, \mathbf{H}_{h}^{n+1}, \boldsymbol{\Lambda}_{h}^{n+1}\right) \in \mathbf{V}_{h} \times \mathbf{V}_{h} \times \mathbf{M}_{h}$ such as

$$
\begin{aligned}
\frac{2 \varepsilon}{\Delta t}\left(\mathbf{E}_{h}^{n+1}, \mathbf{v}\right)_{\mathcal{T}_{h}}-\left(\mathbf{H}_{h}^{n+1}, \mathbf{c u r l} \mathbf{v}\right)_{\mathcal{T}_{h}}= & -\left\langle\boldsymbol{\Lambda}_{h}^{n+1}, \mathbf{n} \times \mathbf{v}\right\rangle_{\partial \mathcal{T}_{h}}+\frac{2 \varepsilon}{\Delta t}\left(\mathbf{E}^{n}, \mathbf{v}\right)_{\mathcal{T}_{h}} \\
& +\left(\mathbf{H}_{h}^{n}, \mathbf{c u r l} \mathbf{v}\right)_{\mathcal{T}_{h}}-\left\langle\boldsymbol{\Lambda}_{h}^{n}, \mathbf{n} \times \mathbf{v}\right\rangle_{\partial \mathcal{T}_{h}} \\
\frac{2 \mu}{\Delta t}\left(\mathbf{H}_{h}^{n+1}, \mathbf{v}\right)_{\mathcal{T}_{h}}+\left(\operatorname{curl} \mathbf{E}_{h}^{n+1}, \mathbf{v}\right)_{\mathcal{T}_{h}}= & -\left\langle\tau \mathbf{n} \times\left(\mathbf{H}_{h}^{n+1}-\boldsymbol{\Lambda}_{h}^{n+1}\right), \mathbf{n} \times \mathbf{v}\right\rangle_{\partial \mathcal{T}_{h}} \\
& +\frac{2 \mu}{\Delta t}\left(\mathbf{H}_{h}^{n}, \mathbf{v}\right)_{\mathcal{T}_{h}}-\left(\mathbf{c u r l} \mathbf{E}_{h}^{n}, \mathbf{v}\right)_{\mathcal{T}_{h}} \\
& -\left\langle\tau \mathbf{n} \times\left(\mathbf{H}_{h}^{n}-\boldsymbol{\Lambda}_{h}^{n}\right), \mathbf{n} \times \mathbf{v}\right\rangle_{\partial \mathcal{T}_{h}}, \\
\frac{2 \gamma}{\Delta t}\left(\boldsymbol{\Lambda}_{h}^{n+1}-\boldsymbol{\Lambda}_{h}^{n}\right)+\left\langle\mathbf{n} \times \mathbf{E}_{h}^{n+1}, \boldsymbol{\eta}\right\rangle_{\partial \mathcal{T}_{h}}= & \left\langle\tau\left(\left(\mathbf{H}_{h}^{t}\right)^{n+1}-\boldsymbol{\Lambda}_{h}^{n+1}\right), \boldsymbol{\eta}\right\rangle_{\partial \mathcal{T}_{h}} \\
& +\left\langle\boldsymbol{\Lambda}_{h}^{n+1}, \boldsymbol{\eta}\right\rangle_{\Gamma_{a}}+\left\langle\mathbf{g}^{\mathrm{inc}, n+1}, \boldsymbol{\eta}\right\rangle_{\Gamma_{a}} \\
& -\left\langle\tau\left(\left(\mathbf{H}_{h}^{t}\right)^{n}-\boldsymbol{\Lambda}_{h}^{n}\right), \boldsymbol{\eta}\right\rangle_{\partial \mathcal{T}_{h}} \\
& -\left\langle\mathbf{n} \times \mathbf{E}_{h}^{n}, \boldsymbol{\eta}\right\rangle_{\partial \mathcal{T}_{h}} \\
& +\left\langle\boldsymbol{\Lambda}_{h}^{n}, \boldsymbol{\eta}\right\rangle_{\Gamma_{a}}+\left\langle\mathbf{g}^{\mathrm{inc}, n}, \boldsymbol{\eta}\right\rangle_{\Gamma_{a}}
\end{aligned}
$$

In order to simplify the equations, we introduce $\bar{\varepsilon}=2 \varepsilon / \Delta t, \bar{\mu}=2 \mu / \Delta t$ and

$$
\begin{aligned}
\mathbf{b}_{E} & =\frac{2 \varepsilon}{\Delta t}\left(\mathbf{E}_{h}^{n}, \mathbf{v}\right)_{\mathcal{T}_{h}}+\left(\mathbf{H}_{h}^{n}, \mathbf{c u r l} \mathbf{v}\right)_{\mathcal{T}_{h}}-\left\langle\boldsymbol{\Lambda}_{h}^{n}, \mathbf{n} \times \mathbf{v}\right\rangle_{\partial \mathcal{T}_{h}}, \\
\mathbf{b}_{H} & =\frac{2 \mu}{\Delta t}\left(\mathbf{H}_{h}^{n}, \mathbf{v}\right)_{\mathcal{T}_{h}}-\left(\operatorname{curl} \mathbf{E}_{h}^{n}, \mathbf{v}\right)_{\mathcal{T}_{h}}-\left\langle\tau \mathbf{n} \times\left(\mathbf{H}_{h}^{n}-\boldsymbol{\Lambda}_{h}^{n}\right), \mathbf{n} \times \mathbf{v}\right\rangle_{\partial \mathcal{T}_{h}}, \\
\mathbf{b}_{\Lambda} & =-\left\langle\mathbf{n} \times \mathbf{E}_{h}^{n}, \boldsymbol{\eta}\right\rangle_{\partial \mathcal{T}_{h}}-\left\langle\tau\left(\left(\mathbf{H}_{h}^{t}\right)^{n}-\boldsymbol{\Lambda}_{h}^{n}\right), \boldsymbol{\eta}\right\rangle_{\partial \mathcal{T}_{h}}+\left\langle\boldsymbol{\Lambda}_{h}^{n}+\mathbf{g}^{\mathrm{inc}, n}+\mathbf{g}^{\mathrm{inc}, n+1}, \boldsymbol{\eta}\right\rangle_{\Gamma_{a}} .
\end{aligned}
$$

The system to be solved can be written as

$$
\begin{aligned}
& \left(\bar{\varepsilon} \mathbf{E}_{h}^{n+1}, \mathbf{v}\right)_{\mathcal{T}_{h}}-\left(\mathbf{H}_{h}^{n+1}, \mathbf{c u r l} \mathbf{v}\right)_{\mathcal{T}_{h}}+\left\langle\Lambda_{h}^{n+1}, \mathbf{n} \times \mathbf{v}\right\rangle_{\partial \mathcal{T}_{h}}=\mathbf{b}_{E},(15 \\
& \left(\bar{\mu} \mathbf{H}_{h}^{n+1}, \mathbf{v}\right)_{\mathcal{T}_{h}}+\left(\operatorname{curl} \mathbf{E}_{h}^{n+1}, \mathbf{v}\right)_{\mathcal{T}_{h}}+\left\langle\tau \mathbf{n} \times\left(\mathbf{H}_{h}^{n+1}-\Lambda_{h}^{n+1}\right), \mathbf{n} \times \mathbf{v}\right\rangle_{\partial \mathcal{T}_{h}}=\mathbf{b}_{H},(16) \\
& \frac{2 \gamma}{\Delta t}\left(\boldsymbol{\Lambda}_{h}^{n+1}-\boldsymbol{\Lambda}_{h}^{n}\right)+\left\langle\mathbf{n} \times \mathbf{E}_{h}^{n+1}, \boldsymbol{\eta}\right\rangle_{\partial \mathcal{T}_{h}}+\left\langle\tau\left(\left(\mathbf{H}_{h}^{t}\right)^{n+1}-\boldsymbol{\Lambda}_{h}^{n+1}\right), \boldsymbol{\eta}\right\rangle_{\partial \mathcal{T}_{h}}-\left\langle\boldsymbol{\Lambda}_{h}^{n+1}, \boldsymbol{\eta}\right\rangle_{\Gamma_{a}}=\mathbf{b}_{\Lambda} \cdot(17
\end{aligned}
$$

By the DAE theory $\gamma$ tends to zero such as we can define the limit

$$
\lim _{\gamma \rightarrow 0} \frac{2 \gamma}{\Delta t}\left(\boldsymbol{\Lambda}_{h}^{n+1}-\boldsymbol{\Lambda}_{h}^{n}\right)=0 .
$$


Then, the system of equations is finally given by (in order to simplify the equations, we omit the superscript ${ }^{n+1}$ )

$$
\begin{aligned}
\left(\bar{\varepsilon} \mathbf{E}_{h}, \mathbf{v}\right)_{\mathcal{T}_{h}}-\left(\mathbf{H}_{h}, \mathbf{c u r l} \mathbf{v}\right)_{\mathcal{T}_{h}}+\left\langle\boldsymbol{\Lambda}_{h}, \mathbf{n} \times \mathbf{v}\right\rangle_{\partial \mathcal{T}_{h}} & =\mathbf{b}_{E}, \\
\left(\bar{\mu} \mathbf{H}_{h}, \mathbf{v}\right)_{\mathcal{T}_{h}}+\left(\mathbf{c u r l} \mathbf{E}_{h}, \mathbf{v}\right)_{\mathcal{T}_{h}}+\left\langle\tau \mathbf{n} \times\left(\mathbf{H}_{h}-\boldsymbol{\Lambda}_{h}\right), \mathbf{n} \times \mathbf{v}\right\rangle_{\partial \mathcal{T}_{h}} & =\mathbf{b}_{H}, \\
\left\langle\mathbf{n} \times \mathbf{E}_{h}, \boldsymbol{\eta}\right\rangle_{\partial \mathcal{T}_{h}}+\left\langle\tau\left(\left(\mathbf{H}_{h}^{t}\right)-\boldsymbol{\Lambda}_{h}\right), \boldsymbol{\eta}\right\rangle_{\partial \mathcal{T}_{h}}-\left\langle\boldsymbol{\Lambda}_{h}, \boldsymbol{\eta}\right\rangle_{\Gamma_{a}} & =\mathbf{b}_{\Lambda} .
\end{aligned}
$$

\subsection{Well-posedness of the local solver}

For $\alpha \in \mathbf{M}_{h},\left(\mathbf{E}_{h}^{\alpha}, \mathbf{H}_{h}^{\alpha}\right)$ denotes the approximate solution at time $n+1$ whose restriction to an element $K \in \mathcal{T}_{h}$ is the solution to the local problem

$$
\begin{aligned}
\left(\bar{\varepsilon} \mathbf{E}_{h}^{\alpha}, \mathbf{v}\right)_{K}-\left(\mathbf{H}_{h}^{\alpha}, \mathbf{c u r l} \mathbf{v}\right)_{K}+\langle\alpha, \mathbf{n} \times \mathbf{v}\rangle_{\partial K} & =\mathbf{b}_{E}, \forall \mathbf{v} \in \mathbf{V}_{h} \\
\left(\bar{\mu} \mathbf{H}_{h}^{\alpha}, \mathbf{v}\right)_{K}+\left(\mathbf{c u r l} \mathbf{E}_{h}^{\alpha}, \mathbf{v}\right)_{K}+\left\langle\tau \mathbf{n} \times\left(\mathbf{H}_{h}^{\alpha}-\alpha\right), \mathbf{n} \times \mathbf{v}\right\rangle_{\partial K} & =\mathbf{b}_{H}, \forall \mathbf{v} \in \mathbf{V}_{h} .
\end{aligned}
$$

Setting $\mathbf{b}_{E}=\mathbf{b}_{H}=0, \mathbf{v}=\mathbf{E}_{h}^{\alpha}$ in (21) and $\mathbf{v}=\mathbf{H}_{h}^{\alpha}$ in (22), from the second equation, we have

$$
\left(\operatorname{curl} \mathbf{E}_{h}^{\alpha}, \mathbf{H}_{h}^{\alpha}\right)_{K}=-\left\langle\tau \mathbf{n} \times\left(\mathbf{H}_{h}^{\alpha}-\alpha\right), \mathbf{n} \times \mathbf{H}_{h}^{\alpha}\right\rangle_{\partial K}-\left(\bar{\mu} \mathbf{H}_{h}^{\alpha}, \mathbf{H}_{h}^{\alpha}\right)_{K},
$$

which is inserted in the first equation and obtain

$$
\left(\bar{\varepsilon} \mathbf{E}_{h}^{\alpha}, \mathbf{E}_{h}^{\alpha}\right)_{K}+\left(\bar{\mu} \mathbf{H}_{h}^{\alpha}, \mathbf{H}_{h}^{\alpha}\right)_{K}+\left\langle\tau \mathbf{n} \times\left(\mathbf{H}_{h}^{\alpha}-\alpha\right), \mathbf{n} \times \mathbf{H}_{h}^{\alpha}\right\rangle_{\partial K}+\left\langle\alpha, \mathbf{n} \times \mathbf{E}_{h}^{\alpha}\right\rangle_{\partial K}=0 .
$$

For $\alpha=0$ and assuming $\tau>0,(23)$ implies that $\mathbf{E}_{h}^{\alpha}=\mathbf{H}_{h}^{\alpha}=0$ on $K$ and since $\bar{\varepsilon}$ and $\bar{\mu}$ are strictly positive real numbers.

\subsection{Characterization of the reduced problem}

Firstly, we consider equation (18) with $\boldsymbol{\Lambda}_{h}=\eta\left(\mathbf{E}_{h}^{\eta}, \mathbf{H}_{h}^{\eta}\right)$ and with $\mathbf{v}=\mathbf{E}_{h}^{\alpha}$ we obtain

$$
\left(\bar{\varepsilon} \mathbf{E}_{h}^{\eta}, \mathbf{E}_{h}^{\alpha}\right)_{\mathcal{T}_{h}}-\left(\mathbf{H}_{h}^{\eta}, \operatorname{curl} \mathbf{E}_{h}^{\alpha}\right)_{\mathcal{T}_{h}}+\left\langle\boldsymbol{\eta}, \mathbf{n} \times \mathbf{E}_{h}^{\alpha}\right\rangle_{\partial \mathcal{T}_{h}}=\mathbf{b}_{E}
$$

Secondly, we set $\mathbf{v}=\mathbf{H}_{h}^{\text {eta }}$ in (19) to obtain

$$
\left(\bar{\mu} \mathbf{H}_{h}^{\alpha}, \mathbf{H}_{h}^{\boldsymbol{\eta}}\right)_{\mathcal{T}_{h}}+\left(\operatorname{curl} \mathbf{E}_{h}^{\alpha}, \mathbf{H}_{h}^{\boldsymbol{\eta}}\right)_{\mathcal{T}_{h}}+\left\langle\tau \mathbf{n} \times\left(\mathbf{H}_{h}^{\alpha}-\alpha\right), \mathbf{n} \times \mathbf{H}_{h}^{\eta}\right\rangle_{\partial \mathcal{T}_{h}}=\mathbf{b}_{H} .
$$

Adding (24) with (25) leads to

$$
\begin{aligned}
\left(\bar{\varepsilon} \mathbf{E}_{h}^{\eta}, \mathbf{E}_{h}^{\alpha}\right)_{\mathcal{T}_{h}}+\left(\bar{\mu} \mathbf{H}_{h}^{\alpha}, \mathbf{H}_{h}^{\eta}\right)_{\mathcal{T}_{h}} & +\left\langle\eta, \mathbf{n} \times \mathbf{E}_{h}^{\alpha}\right\rangle_{\partial \mathcal{T}_{h}} \\
& +\left\langle\tau \mathbf{n} \times\left(\mathbf{H}_{h}^{\alpha}-\alpha\right), \mathbf{n} \times \mathbf{H}_{h}^{\eta}\right\rangle_{\partial \mathcal{T}_{h}}=\mathbf{b}_{E}+\mathbf{b}_{H} .
\end{aligned}
$$


The conservativity condition (20) for $\alpha \in \mathbf{M}_{h}$ where $\left(\mathbf{E}_{h}^{\alpha}, \mathbf{H}_{h}^{\alpha}\right)$ denotes the approximate solution, yields

$$
\left\langle\mathbf{n} \times \mathbf{E}_{h}^{\alpha}, \boldsymbol{\eta}\right\rangle_{\partial \mathcal{T}_{h}}+\left\langle\tau \mathbf{n} \times\left(\mathbf{H}_{h}^{\alpha}-\alpha\right), \mathbf{n} \times \boldsymbol{\eta}\right\rangle_{\partial \mathcal{T}_{h}}-\langle\alpha, \boldsymbol{\eta}\rangle_{\Gamma_{a}}=\mathbf{b}_{\Lambda}
$$

We replace $\left\langle\mathbf{n} \times \mathbf{E}_{h}^{\alpha}, \boldsymbol{\eta}\right\rangle_{\partial \mathcal{T}_{h}}$ in (26) by (27) and we deduce that $\alpha$ is the solution of the following reduced problem for $\alpha$

$$
\mathcal{A}_{h}(\alpha, \eta)=\mathcal{L}_{h}(\eta), \forall \eta \in \mathbf{M}_{h}
$$

with

$$
\begin{aligned}
& \mathcal{A}_{h}(\alpha, \eta)=\left(\bar{\varepsilon} \mathbf{E}_{h}^{\alpha}, \mathbf{E}_{h}^{\eta}\right)_{\mathcal{T}_{h}}+\left(\bar{\mu} \mathbf{H}_{h}^{\alpha}, \mathbf{H}_{h}^{\eta}\right)_{\mathcal{T}_{h}}+\left\langle\tau \mathbf{n} \times\left(\alpha-\mathbf{H}_{h}^{\alpha}\right), \mathbf{n} \times\left(\eta-\mathbf{H}_{h}^{\eta}\right)\right\rangle_{\partial \mathcal{T}_{h}}+\langle\alpha, \boldsymbol{\eta}\rangle_{\Gamma_{a}} \\
& \mathcal{L}_{h}(\eta)=\mathbf{b}_{E}+\mathbf{b}_{H}-\mathbf{b}_{\Lambda} .
\end{aligned}
$$

Note that the bilinear form $\mathcal{A}_{h}$ is symmetric positive definite. Let us now prove the existence and uniqueness of the approximate solution.

Theorem 1. If we assume $\tau>0$, then the HDG method (18)-(20) has a unique solution $\left(\mathbf{E}_{h}, \mathbf{H}_{h}, \boldsymbol{\Lambda}_{h}\right)$ for any time iteration $n \geq 1$.

Proof. We suppose that the right-hand side of (28) is equal to zero

$$
\mathcal{A}_{h}(\alpha, \eta)=0, \forall \eta \in \mathbf{M}_{h}
$$

Next, we choose $\eta=\alpha$ and the relation becomes

$$
\mathcal{A}_{h}(\alpha, \alpha)=\bar{\varepsilon}\left\|\mathbf{E}_{h}^{\alpha}\right\|^{2}+\bar{\mu}\left\|\mathbf{H}_{h}^{\alpha}\right\|^{2}+\left\langle\tau \mathbf{n} \times\left(\alpha-\mathbf{H}_{h}^{\alpha}\right), \mathbf{n} \times\left(\alpha-\mathbf{H}_{h}^{\alpha}\right)\right\rangle_{\partial \mathcal{T}_{h}}+\langle\alpha, \alpha\rangle_{\Gamma_{a}} .
$$

A sufficient condition for having $\mathcal{A}_{h}(\alpha, \alpha)=0$ is to require that each of the terms are zero. The two first terms imply that $\mathbf{E}_{h}^{\alpha}=\mathbf{H}_{h}^{\alpha}=0$ for all $K \in \mathcal{T}_{h}$ since $\bar{\varepsilon}>0$ and $\bar{\mu}>0$. Moreover, $\alpha=0$ on $\Gamma_{a}$ by $\langle\alpha, \alpha\rangle_{\Gamma_{a}}=0$. Then, from $\mathbf{H}_{h}^{\alpha}=0$, we have

$$
\left\langle\tau \mathbf{n} \times\left(\alpha-\mathbf{H}_{h}^{\alpha}\right), \mathbf{n} \times\left(\alpha-\mathbf{H}_{h}^{\alpha}\right)\right\rangle_{\partial \mathcal{T}_{h}}=\langle\tau \mathbf{n} \times \alpha, \mathbf{n} \times \alpha\rangle_{\partial \mathcal{T}_{h}}
$$

Assuming $\tau>0$ implies that $\alpha=0$ on $\partial K$, for all $K \in \mathcal{T}_{h}$. In summary, $\alpha=0$ on $\mathcal{F}_{h} \cap \Gamma_{a}$ and on $\mathcal{T}_{h}$, so $\alpha=0$ everywhere. 


\subsection{Energy variation and unconditional stability}

The total discrete electromagnetic energy in $\mathcal{T}_{h}$ at time $n$ is given by

$$
\mathcal{E}^{n}=\frac{1}{2}\left(\bar{\varepsilon}|| \mathbf{E}_{h}^{n}\left\|^{2}+\bar{\mu}\right\| \mathbf{H}_{h}^{n} \|^{2}\right) \text {. }
$$

Lemma 1. Using (15)-(17), the total discrete electromagnetic energy defined by (29) is non-increasing in time i.e. $\mathcal{E}^{n+1} \leq \mathcal{E}^{n}$. Then, the totally discretized problem (18)(20) is unconditionally stable.

Proof. Choosing as test functions $\mathbf{v}=\left(\mathbf{E}_{h}^{n+1}+\mathbf{E}_{h}^{n}\right) / 2$ belonging to $\mathbf{V}_{h}$ in (15) and $\mathbf{v}=\left(\mathbf{H}_{h}^{n+1}+\mathbf{H}_{h}^{n}\right) / 2$ belonging to $\mathbf{V}_{h}$ in (16) and summing

$$
\begin{aligned}
& \frac{1}{2}\left(\bar{\varepsilon}\left\|\mathbf{E}_{h}^{n+1}\right\|^{2}+\bar{\mu}\left\|\mathbf{H}_{h}^{n+1}\right\|^{2}\right)+\left\langle\boldsymbol{\Lambda}_{h}^{n+1}, \mathbf{n} \times\left(\frac{\mathbf{E}_{h}^{n+1}+\mathbf{E}_{h}^{n}}{2}\right)\right\rangle_{\partial \mathcal{T}_{h}} \\
& +\left\langle\tau \mathbf{n} \times\left(\mathbf{H}_{h}^{n+1}-\boldsymbol{\Lambda}_{h}^{n+1}\right), \mathbf{n} \times\left(\frac{\mathbf{H}_{h}^{n+1}+\mathbf{H}_{h}^{n}}{2}\right)\right\rangle_{\partial \mathcal{T}_{h}} \\
= & \frac{1}{2}\left(\bar{\varepsilon}\left\|\mathbf{E}_{h}^{n}\right\|^{2}+\bar{\mu}\left\|\mathbf{H}_{h}^{n}\right\|^{2}\right)-\left\langle\boldsymbol{\Lambda}_{h}^{n}, \mathbf{n} \times\left(\frac{\mathbf{E}_{h}^{n+1}+\mathbf{E}_{h}^{n}}{2}\right)\right\rangle_{\partial \mathcal{T}_{h}} \\
& -\left\langle\tau \mathbf{n} \times\left(\mathbf{H}_{h}^{n}-\boldsymbol{\Lambda}_{h}^{n}\right), \mathbf{n} \times\left(\frac{\mathbf{H}_{h}^{n+1}+\mathbf{H}_{h}^{n}}{2}\right)\right\rangle_{\partial \mathcal{T}_{h}} .
\end{aligned}
$$

The energy variation is then given by

$$
\begin{aligned}
\mathcal{E}^{n+1}-\mathcal{E}^{n}= & -\left\langle\left(\boldsymbol{\Lambda}_{h}^{n+1}+\boldsymbol{\Lambda}_{h}^{n}\right), \mathbf{n} \times\left(\frac{\mathbf{E}_{h}^{n+1}+\mathbf{E}_{h}^{n}}{2}\right)\right\rangle_{\partial \mathcal{T}_{h}} \\
& -\left\langle\tau \mathbf{n} \times\left(\mathbf{H}_{h}^{n+1}+\mathbf{H}_{h}^{n}\right), \mathbf{n} \times\left(\frac{\mathbf{H}_{h}^{n+1}+\mathbf{H}_{h}^{n}}{2}\right)\right\rangle_{\partial \mathcal{T}_{h}} \\
& +\left\langle\tau \mathbf{n} \times\left(\boldsymbol{\Lambda}_{h}^{n+1}+\boldsymbol{\Lambda}_{h}^{n}\right), \mathbf{n} \times\left(\frac{\mathbf{H}_{h}^{n+1}+\mathbf{H}_{h}^{n}}{2}\right)\right\rangle_{\partial \mathcal{T}_{h}}
\end{aligned}
$$

From (17) with $\eta=\boldsymbol{\Lambda}_{h}=\boldsymbol{\Lambda}_{h}^{n+1}+\boldsymbol{\Lambda}_{h}^{n}, \mathbf{E}_{h}=\left(\mathbf{E}_{h}^{n+1}+\mathbf{E}_{h}^{n}\right) / 2$ and $\mathbf{H}_{h}=\left(\mathbf{H}_{h}^{n+1}+\mathbf{H}_{h}^{n}\right) / 2$, we have

$$
\begin{aligned}
\left\langle\mathbf{n} \times \frac{\mathbf{E}_{h}^{n+1}+\mathbf{E}_{h}^{n}}{2}, \boldsymbol{\Lambda}_{h}^{n+1}+\boldsymbol{\Lambda}_{h}^{n}\right\rangle_{\partial \mathcal{T}_{h}}= & -\left\langle\tau \mathbf{n} \times\left(\mathbf{H}_{h}^{n+1}+\mathbf{H}_{h}^{n}\right), \mathbf{n} \times\left(\frac{\mathbf{H}_{h}^{n+1}+\mathbf{H}_{h}^{n}}{2}\right)\right\rangle_{\partial \mathcal{T}_{h}} \\
& +\left\|\boldsymbol{\Lambda}_{h}^{n+1}+\boldsymbol{\Lambda}_{h}^{n}\right\|^{2} .
\end{aligned}
$$


Finally, we found the result

$$
\mathcal{E}^{n+1}-\mathcal{E}^{n}=-\left\langle\tau \mathbf{n} \times\left(\mathbf{H}_{h}^{n+1}+\mathbf{H}_{h}^{n}\right), \mathbf{n} \times\left(\frac{\mathbf{H}_{h}^{n+1}+\mathbf{H}_{h}^{n}}{2}\right)\right\rangle_{\partial \mathcal{T}_{h}}-\left\|\boldsymbol{\Lambda}_{h}^{n+1}+\boldsymbol{\Lambda}_{h}^{n}\right\|^{2} \leq 0 .
$$

\section{HDG method implementation}

Let us introduce some notations and definitions. For all $K_{e} \in \mathcal{T}_{h}$ we have

$$
\left(\left.\mathbf{E}_{h}\right|_{K_{e}},\left.\mathbf{H}_{h}\right|_{K_{e}}\right)=\left(\mathbf{E}^{e}, \mathbf{H}^{e}\right): K_{e} \times K_{e} \longrightarrow \mathbb{R}^{3} \times \mathbb{R}^{3},
$$

where $\mathbf{E}^{e}(\mathbf{x})=\left[E_{x}^{e}(\mathbf{x}), E_{y}^{e}(\mathbf{x}), E_{z}^{e}(\mathbf{x})\right]^{T}$ and $\mathbf{H}^{e}(\mathbf{x})=\left[H_{x}^{e}(\mathbf{x}), H_{y}^{e}(\mathbf{x}), H_{z}^{e}(\mathbf{x})\right]^{T}$. The components of electromagnetic field are expanded in element $K_{e}$ by a linear combination of basis functions $\varphi_{j}^{e}(\mathbf{x}) \subset \mathbb{P}_{p_{e}}\left(K_{e}\right)$

$$
E_{\xi}^{e}(\mathbf{x})=\sum_{j=1}^{N_{K}^{e}} \underline{E}_{\xi}^{e}[j] \varphi_{j}^{e}(\mathbf{x}), \quad H_{\xi}^{e}(\mathbf{x})=\sum_{j=1}^{N_{K}^{e}} \underline{H}_{\xi}^{e}[j] \varphi_{j}^{e}(\mathbf{x}) \quad(\xi \in\{x, y, z\}),
$$

where $N_{K}^{e}=\left(p_{e}+1\right)\left(p_{e}+2\right)\left(p_{e}+3\right) / 6$ the dimension of $\mathbb{P}_{p_{e}}, \underline{E}_{\xi}^{e}[j]$ and $\underline{H}_{\xi}^{e}[j]$ are the DoFs of the electromagnetic field supporting by $K_{e}$. For all $F_{f} \in \mathcal{F}_{h}$, we have

$$
\begin{aligned}
\boldsymbol{\Lambda}_{\left.h\right|_{F_{f}}=} \boldsymbol{\Lambda}^{f} & : F_{f} \longrightarrow \mathbb{R}^{3} \\
\boldsymbol{\Lambda}^{f} & :(\mathbf{x}) \longmapsto \Lambda_{\mathbf{u}}^{f}(\mathbf{x}) \mathbf{u}^{f}+\Lambda_{\mathbf{w}}^{f}(\mathbf{x}) \mathbf{w}^{f},
\end{aligned}
$$

where $\mathbf{u}^{f}$ and $\mathbf{w}^{f}$ represent coordinate axis. $\Lambda_{\mathbf{u}}^{f}$ and $\Lambda_{\mathbf{w}}^{f}$ are decomposed by linear combinations of basis functions $\psi_{j}^{f}(\mathbf{x}) \subset \mathbb{P}_{p_{f}}\left(F_{f}\right)$, such as

$$
\Lambda_{\mathbf{u}}^{f}(\mathbf{x})=\sum_{j=1}^{N_{F}^{f}} \underline{\Lambda}_{\mathbf{u}}^{f}[j] \psi_{j}^{f}(\mathbf{x}), \quad \Lambda_{\mathbf{w}}^{f}(\mathbf{x})=\sum_{j=1}^{N_{F}^{f}} \underline{\Lambda}_{\mathbf{w}}^{f}[j] \psi_{j}^{f}(\mathbf{x}),
$$

where $\underline{\Lambda}_{\mathbf{u}}^{f}[j]$ and $\underline{\Lambda}_{\mathbf{w}}^{f}[j]$ are the DoFs of $\Lambda^{f}$ associated to $F_{f}$, and $N_{F}^{f}=\left(p_{f}+1\right)\left(p_{f}+2\right) / 2$ the dimension of $\mathbb{P}_{p_{f}}\left(F_{f}\right)$.

Remark 3. In practice, $\mathbf{u}^{f}=\overline{\mathbf{u}}^{f} /\left\|\overline{\mathbf{u}}^{f}\right\|_{2}$ and $\mathbf{w}^{f}=\overline{\mathbf{w}}^{f} /\left\|\overline{\mathbf{w}}^{f}\right\|_{2}$ with $\overline{\mathbf{u}}^{f}=n_{2}^{f}-n_{1}^{f}$ and $\overline{\mathbf{w}}^{f}=n_{3}^{f}-n_{1}^{f}$ where $n_{1}^{f}, n_{2}^{f}$ and $n_{3}^{f}$ represent the three nodes associated to $F_{f}$. 
Henceforth, we denote by $\nu_{e}$ the set of indices of neighboring elements of $K_{e}$. For all $K_{e} \in \mathcal{T}_{h}\left(e \in\left\{1, \cdots,\left|\mathcal{T}_{h}\right|\right)\right.$, we have $\left|\nu_{e}\right|$ faces, denoted by $\partial K_{e}^{l} \in \partial \mathcal{T}_{h}$ and defined by

$$
\partial K_{e}^{l}=\overline{K_{e}} \cap \overline{K_{g}}, \quad l \in\left\{1, \cdots,\left|\nu_{e}\right|\right\}, \quad g \in \nu_{e} .
$$

We get a local indexing of faces, element per element. Moreover, we define the function $\sigma$ that maps the local to the global numerotation in $\mathcal{F}_{h}$ (see Figure 1)

$$
\left\{\begin{array}{l}
\forall F_{f} \in \mathcal{F}_{h}^{I} \text { such that } F_{f}=\partial K_{e}^{l} \cap \partial K_{g}^{k}, \quad \sigma(e, l)=\sigma(g, k)=f, \\
\forall F_{f} \in \mathcal{F}_{h}^{B} \text { such that } F_{f}=\partial K_{e}^{l} \cap\left(\Gamma_{m} \cup \Gamma_{m}\right), \quad \sigma(e, l)=f .
\end{array}\right.
$$

In order to approach the values of $\left(\mathbf{E}_{h}, \mathbf{H}_{h)}\right.$ in an element $K_{e} \in \mathcal{T}_{h}$, one has to know
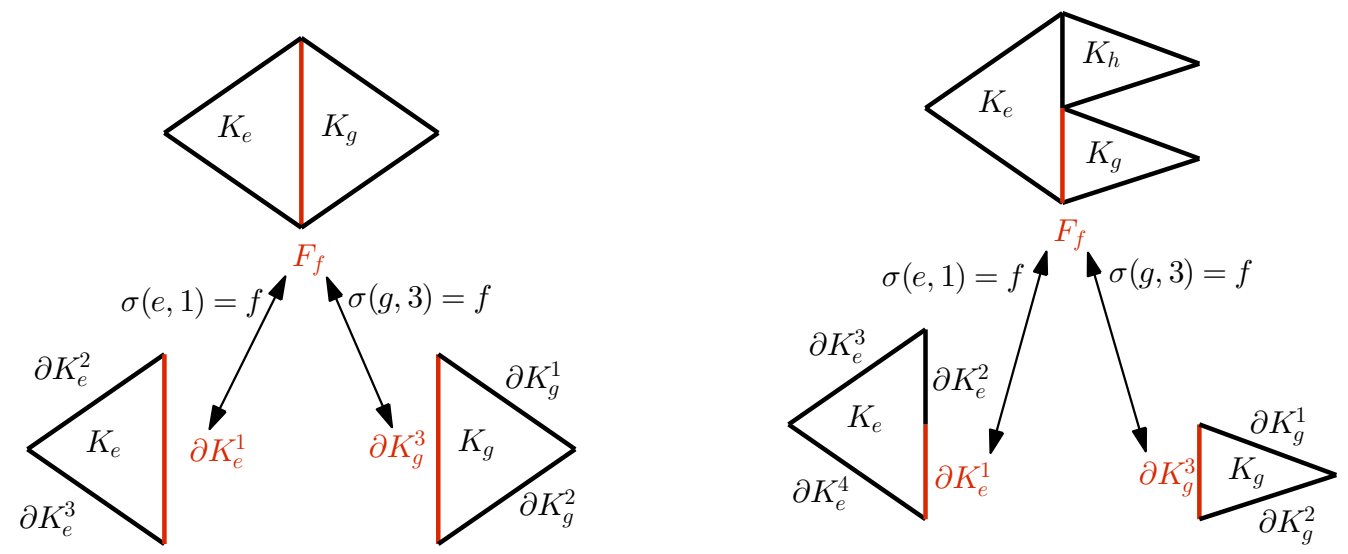

Figure 1: Function $\sigma$ and indexing (local/global) in 2D: conforming mesh (left) and non-conforming mesh (right).

the values of the fields in each neighboring element of $K_{e}$. One major criticism of DG method is that they have too many degrees of freedom (DoFs) due to nodal duplication at the element boundary interfaces

$$
\sum_{e=1}^{\left|\mathcal{T}_{h}\right|} 6 N_{K}^{e}
$$

where $N_{K}^{e}$ is the dimension of $\mathbb{P}_{p_{e}}\left(K_{e}\right)$, i.e. $N_{K}^{e}=\left(p_{e}+1\right)\left(p_{e}+2\right)\left(p_{e}+3\right) / 6$. The goal of the HDG method is to reduce the number of globally coupled DoFs. For that purpose, the HDG method introduces a hybrid variable, as a new unknown, on the faces of $\mathcal{F}_{h}$. This new unknown can be determined by solving a global linear 
system from the discretization of a conservation condition for the hybrid variable. More exactly, the DoFs from the hybrid variable can be defined by the resolution of a global linear system. When the DoFs of the hybrid variable are known, the electromagnetic field $\left(\mathbf{E}_{h}, \mathbf{H}_{h}\right)$ can be locally determined element wise thanks to the resolution of local linear systems defined in each $K \in \mathcal{T}_{h}$. For the HDG method, the total number of globally coupled DoFs is given by

$$
\sum_{f=1}^{\left|\mathcal{F}_{h}\right|} 2 N_{F}^{f}
$$

where

$$
N_{F}^{f}=\left(p_{f}+1\right)\left(p_{f}+2\right) / 2,
$$

is the dimension of the space $\mathbb{P}_{p_{f}}\left(F_{f}\right)$.

Remark 4. If we consider a uniform degree of interpolation for elements and faces, i.e. $p_{e}=p_{f}=p, \forall K_{e} \in \mathcal{T}_{h}, \forall F_{f} \in \mathcal{F}_{h}$, then the total numbers of globally coupled DoFs are

DG method: $\quad\left(p+1(p+2)(p+3)\left|\mathcal{T}_{h}\right|\right.$,

HDG method: $\quad(p+1)(p+2)\left|\mathcal{F}_{h}\right|$.

For $\left|\mathcal{F}_{h}\right| \approx 2\left|\mathcal{T}_{h}\right|, 2 /(p+3)$ is the ratio of the number of globally coupled DoFs of HDG method to DG method.

\subsection{Local linear system}

Taking (30)-(31) and choosing the basis functions of $\mathbf{V}_{h}$ as test functions in (18)(20) we obtain that $\left[\underline{E}_{x}^{e}, \underline{E}_{y}^{e}, \underline{E}_{z}^{e}, \underline{H}_{y}^{e}, \underline{H}_{x}^{e}, \underline{H}_{z}^{e}\right]^{T}$ and $\left[\underline{\Lambda}_{\mathbf{u}}^{\sigma(e, l)}, \underline{\Lambda}_{\mathbf{w}}^{\sigma(e, l)}\right]^{T}, 1 \leq l \leq\left|\nu_{e}\right|$ satisfy the following system

$$
\mathbb{A}^{e}\left[\begin{array}{l}
\underline{E}_{x}^{e} \\
\underline{E}_{y}^{e} \\
\underline{E}_{z}^{e} \\
\underline{H}_{y}^{e} \\
\underline{H}_{x}^{e} \\
\underline{H}_{z}^{e}
\end{array}\right]+\sum_{l=1}^{\left|\nu_{e}\right|} \mathbb{C}^{(e, l)}\left[\begin{array}{l}
\underline{\Lambda}_{\mathbf{u}}^{\sigma(e, l)} \\
\underline{\Lambda}_{\mathbf{w}}^{\sigma(e, l)}
\end{array}\right]=\mathbb{P}^{e}
$$

$\mathbb{A}^{e}, \mathbb{C}^{(e, l)}$, being matrices of dimensions $6 N_{K}^{e} \times 6 N_{K}^{e}$ and $6 N_{K}^{e} \times 2 N_{F}^{\sigma(e, l)}$ and $\mathbb{P}^{e}$ a local right-hand side. 


\subsection{Global linear system}

A global linear system can be obtained for the DoFs of the hybrid variable $\Lambda_{h}$ in $\mathcal{F}_{h}$. Let us start by $F_{f} \in \mathcal{F}_{h}^{I}$ an interior face between two elements $K_{e}$ and $K_{g}$. This face takes the local indices $l$ and $k$ on $K_{e}$ and $K_{g}$, respectively, in particular $f=\sigma(e, l)=\sigma(g, k)$. For all $\boldsymbol{\eta} \in \mathbf{M}_{h}$ we have

$$
\begin{aligned}
\left\langle\mathbf{n} \times \mathbf{E}_{h}, \boldsymbol{\eta}\right\rangle_{\partial K_{e}^{l}} & -\tau^{(e, l)}\left\langle\mathbf{n} \times\left(\mathbf{n} \times \mathbf{H}_{h}\right), \boldsymbol{\eta}\right\rangle_{\partial K_{e}^{l}}-\tau^{(e, l)}\left\langle\boldsymbol{\Lambda}_{h}, \boldsymbol{\eta}\right\rangle_{\partial K_{e}^{l}} \\
& +\left\langle\mathbf{n} \times \mathbf{E}_{h}, \boldsymbol{\eta}\right\rangle_{\partial K_{g}^{k}}-\tau^{(g, k)}\left\langle\mathbf{n} \times\left(\mathbf{n} \times \mathbf{H}_{h}\right), \boldsymbol{\eta}\right\rangle_{\partial K_{g}^{k}} \\
& -\tau^{(g, k)}\left\langle\boldsymbol{\Lambda}_{h}, \boldsymbol{\eta}\right\rangle_{\partial K_{g}^{k}}=0 .
\end{aligned}
$$

Now, we consider a boundary face $F_{f} \in \Gamma_{a}$ such as $F_{f} \in \partial K_{e}^{l} \cap \Gamma_{a}$. For all $\boldsymbol{\eta} \in \mathbf{M}_{h}$, the conservation condition takes the form

$$
\begin{aligned}
\left\langle\mathbf{n} \times \mathbf{E}_{h}, \boldsymbol{\eta}\right\rangle_{\partial K_{e}^{l}}-\tau^{(e, l)}\left\langle\mathbf{n} \times\left(\mathbf{n} \times \mathbf{H}_{h}\right), \boldsymbol{\eta}\right\rangle_{\partial K_{e}^{l}} \\
-\left(1+\tau^{(e, l)}\right)\left\langle\boldsymbol{\Lambda}_{h}, \boldsymbol{\eta}\right\rangle_{\partial K_{e}^{l}}=\left\langle\mathbf{g}^{i n c}, \boldsymbol{\eta}\right\rangle_{\partial K_{e}^{l}} .
\end{aligned}
$$

Using (30)-(31), replacing the test functions in (34) by the basis functions of $\mathbf{M}_{h}$ and using (35) we can find another relation between $\left[\underline{E}_{x}^{e}, \underline{E}_{y}^{e}, \underline{E}_{z}^{e}, \underline{H}_{y}^{e}, \underline{H}_{x}^{e}, \underline{H}_{z}^{e}\right]^{T}$ and $\left[\underline{\Lambda}_{\mathrm{u}}^{\sigma(e, l)}, \underline{\Lambda}_{\mathrm{w}}^{\sigma(e, l)}\right]^{T}, 1 \leq l \leq\left|\nu_{e}\right|$. With (33) we obtain the following system

$$
\mathbb{K} \underline{\Lambda}=\underline{\mathbf{b}_{\Lambda}},
$$

where $\mathbb{K}$ is a matrix of size $\sum_{f=1}^{\left|\mathcal{F}_{h}\right|} 2 N_{F}^{f} \times \sum_{f=1}^{\left|\mathcal{F}_{h}\right|} 2 N_{F}^{f}$ and $\underline{\mathbf{b}_{\Lambda}}$ is a vector of length $\sum_{f=1}^{\left|\mathcal{F}_{h}\right|} 2 N_{F}^{f}$.

\section{Numerical results}

The HDGTD method described in the previous sections has been programmed in Fortran 95/2003 for a renormalized version of the time-domain Maxwell equations (1). The equations are still formulated as in (1) but with $\varepsilon \equiv \varepsilon_{r}$ and $\mu \equiv \mu_{r}$ where $\varepsilon_{r}$ and $\mu_{r}$ are relative electromagnetic parameters without dimension. Beside, in the renormalized equations, the time variable $t$ is given in meter while the electric and magnetic fields, $\mathbf{E}$ and $\mathbf{H}$, are obtained in Volts per meter $(\mathrm{V} / \mathrm{m})$. 


\subsection{Propagation of a standing wave in a cubic PEC cavity}

In order to validate and to study the numerical convergence of the proposed $3 \mathrm{D}$ HDG method, we consider the propagation of an eigenmode in a closed cavity $(\Omega$ is the unit square) where the walls are metallic, the frequency $f=\sqrt{3} / 2 c_{0} \mathrm{~Hz}$, where $c_{0}$ is the speed of light in vacuum, and the wavelength $\lambda=1.1547 \mathrm{~m}$. The permittivity and the permeability are equals to the constant vacuum values $\varepsilon=\varepsilon_{0}$ and $\mu=\mu_{0}$ i.e. $\varepsilon_{r}=\mu_{r}=1$. The exact time-domaine solution is given by

$$
\left\{\begin{array}{rr}
E_{x}(x, y, z, t)= & -\cos (\pi x) \sin (\pi y) \sin (\pi z) \cos (\omega t), \\
E_{y}(x, y, z, t)= & 0, \\
E_{z}(x, y, z, t)= & \sin (\pi x) \sin (\pi y) \cos (\pi z) \cos (\omega t)
\end{array}\right.
$$

and

$$
\left\{\begin{array}{l}
H_{x}(x, y, z, t)=-\frac{\pi}{\omega} \sin (\pi x) \cos (\pi y) \cos (\pi z) \sin (\omega t), \\
H_{y}(x, y, z, t)=\frac{2 \pi}{\omega} \cos (\pi x) \sin (\pi y) \cos (\pi z) \sin (\omega t), \\
H_{z}(x, y, z, t)=-\frac{\pi}{\omega} \cos (\pi x) \cos (\pi y) \sin (\pi z) \sin (\omega t)
\end{array}\right.
$$

where the angular frequency (or pulsation) is given by $\omega=2 \pi f\left(\mathrm{rad} \cdot \mathrm{s}^{-1}\right)$. The above relations are used to initialize the $\mathbf{E}$ and $\mathbf{H}$ field components in the cavity.

The parameter $\tau$ in the HDG traces is taken equal to 1 . The time step is chosen as $\Delta t=c_{\mathrm{CFL}} \Delta t_{\min }$ where $\Delta t_{\min }$ is the global minimal time step over the whole mesh. The value of the time step is chosen such that the accuracy does not affect the accuracy in space. We measure the maximal $L_{2}$-norm of the error for a sequence of successively refined tetrahedral meshes starting from a uniform coarse mesh. The latter is obtained by subdividing a finite difference grid of the unit cube. We plot this error as a function of the square root of the number of DoFs required for the interpolation of the electric and magnetic field, in logarithmic scale. On Figure 2a, we observe an optimal convergence order for the electromagnetic field. For a too large value of the coefficient $c_{\mathrm{CFL}}$, the discretization error in time is predominant and the order is equal to 2 independently of the interpolation degree, see Figure $2 \mathrm{~b}$. We proceed by comparing the proposed time-implicit HDG with a classical centered flux time-implicit DGTD method [24] in terms of memory occupation and $\mathrm{CPU}$ time for the same problem. The coefficient $c_{\mathrm{CFL}}$ is set to 1 . We make use of the MUMPS sparse direct solver [31] for the inversion of the HDG system (36) and of the global linear system characterizing the time-implicit DGDT method. Simulations are performed on a workstation equipped with an Intel Xeon E5-2630@2.60 GHz processsor. The obtained results are summarized in Table 1 for two interpolation 


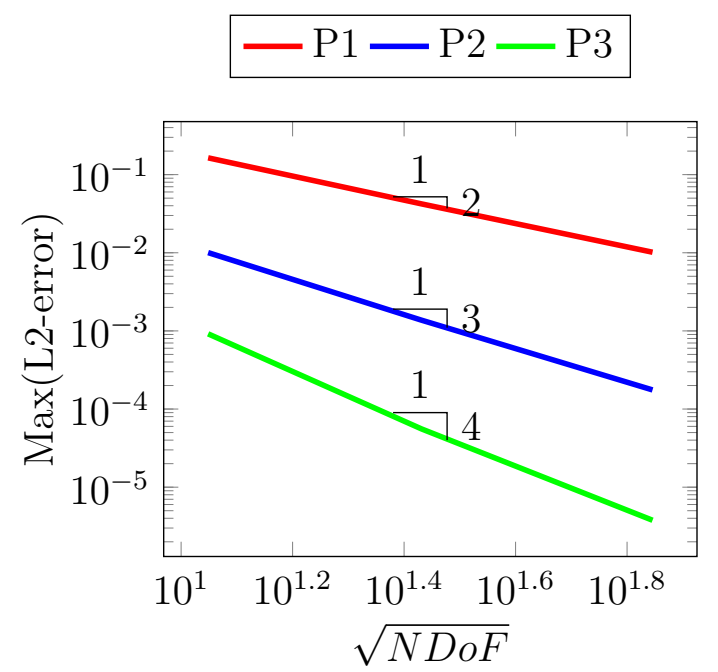

(a) $c_{\mathrm{CFL}}<<1$

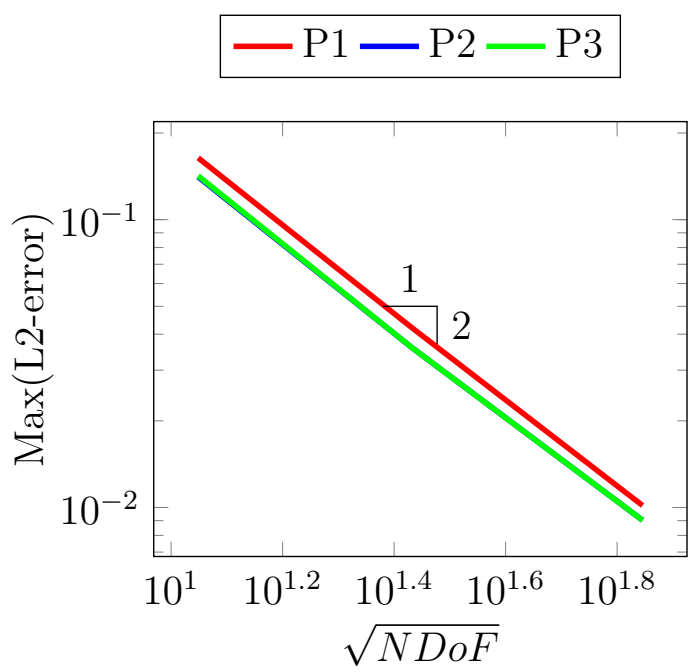

(b) $c_{\mathrm{CFL}}=1$

Figure 2: Standing wave in a PEC cavity: numerical convergence study.

orders. We clearly see that the HDG method outperforms the DG method both on the memory requirement and $\mathrm{CPU}$ time metrics, especially at higher interpolation orders.

\subsection{Scattering of a plane wave by a dielectric sphere}

We now consider the problem of the scattering of a plane wave by a dielectric sphere in free space. The computational domain $\Omega_{1}$ is artificially bounded by a sphere with radius $r_{1}=1.5 \mathrm{~m}$ on which the Silver-Müller absorbing condition is applied. The dielectric sphere $\Omega_{2}$ is assumed to have a radius of $r_{2}=0.5 \mathrm{~m}$ and bounds a material of relative permittivity $\varepsilon_{r, 2}=2$ and relative permeability $\mu_{r, 2}=1$ (non-magnetic material). The medium exterior to the dielectric sphere is assumed to be vacuum, i.e. $\varepsilon_{r, 1}=\mu_{r, 1}=1$. At initial time, the electromagnetic field is set to zero while the incident plane wave is defined by $E_{x}^{\text {inc }}=E_{y}^{\text {inc }}=H_{x}^{\text {inc }}=H_{z}^{\text {inc }}=0$ and

$$
\left\{\begin{array}{l}
E_{z}^{\text {inc }}(x, y, z, t=0)=\cos (\omega y), \\
H_{y}^{\text {inc }}(x, y, z, t=0)=-\cos (\omega y)
\end{array}\right.
$$

We consider two cases. In the first one, the frequency of the incident plane wave is equal to $F=300 \mathrm{MHz}$ and the total simulation time is set to $T=3.3310^{-8} \mathrm{~s}$ which corresponds to a propagation of the initial wave over 10 periods. In this case 


\begin{tabular}{ccccc} 
& \multicolumn{2}{c}{$\mathbb{P}^{1}$} & \multicolumn{2}{c}{$\mathbb{P}^{3}$} \\
& DG & HDG & DG & HDG \\
\hline Matrix order & 36864 & 39168 & 184320 & 130560 \\
\# non-zero entries & 2988320 & 1562112 & 64448080 & 17356800 \\
Fill-in ratio (\%) & 0.21 & 0.1 & 0.19 & 0.1 \\
Storage LU (MB) & 541 & 115 & 13442 & 1271 \\
Overall storage (MB) & 827 & 188 & 20383 & 1985 \\
Analysis time (s) & 0.7 & 0.09 & 12 & 0.7 \\
Factorization time (s) & 46 & 3 & 8308 & 143 \\
$L_{2}$ error & $4.6810^{-2}$ & $4.2210^{-2}$ & $3.6110^{-2}$ & $3.6010^{-2}$ \\
\hline
\end{tabular}

Table 1: Standing wave in a PEC cavity: comparaison between the time-implicit DGTD method and the time-implicit HDGTD method.

the time step is set to $\Delta t=311 \Delta t_{\min }$ where $\Delta t_{\min }$ is the smallest time step over the whole mesh which is equal to $10^{-12} \mathrm{~s}$ (the largest time step is equal to $1.910^{-10} \mathrm{~s}$ ). In the second case, we set $F=600 \mathrm{MHz}$ and a final time $T=1.6710^{-8} \mathrm{~S}$ which also corresponds to a propagation of the initial wave over 10 periods. The CFL number is here set to 155 . We present results for HDG- $\mathbb{P}_{k}$ methods with $k=1,2$,. The better accuracy with increasing $k$ is here limited by the second-order time approximation (as mentioned in the previous case) and by the approximation error on the circular geometry of the interface between the two materials as well as the use of the first order Silver-Müller absorbing condition. The contour lines of the real part of the discrete Fourier transform (DFT) of the component $E_{z}$ evaluated are exposed in Figures 3 and Figures 4, respectively. To assess the performances of the HDG method, we summarize in Table 2 different values, in particular the number of nonzero elements and the memory requirement for the analysis and factorization of the matrix of system (36). These value are here independent of the frequency $F$ on the incident plane wave. Data for a simulation performed with a fully explicit centered flux-based DG method [7] are also given. Despite the overhead introduced by the resolution of the linear system for the hybrid variable (36), the time-implicit HDG method is faster in CPU time, thanks to the possibility of using a larger time step.

\section{Conclusion}

In this work we have presented a time-implicit hybridizable discontinuous Galerkin method to solve numerically the time-domain Maxwell equations in 3D. We have provided preliminary numerical results demonstrating that the method can be com- 


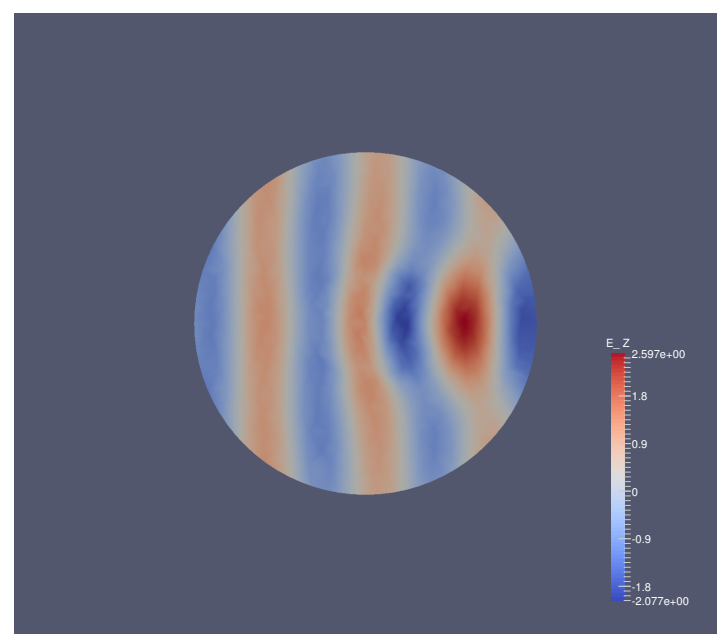

(a) HDG- $\mathbb{P}_{1}$ method.

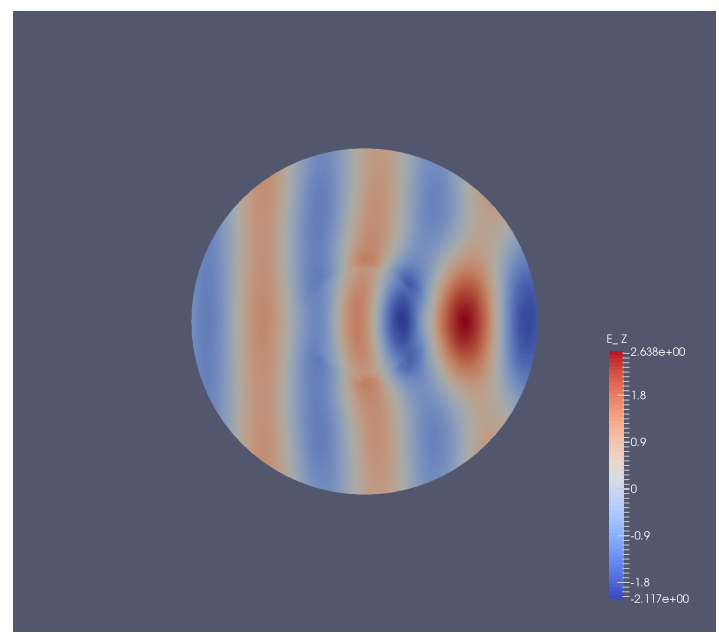

(b) HDG- $\mathbb{P}_{2}$ method.

Figure 3: Scattering of a plane wave by a dielectric sphere: contour lines of $\operatorname{DFT}\left(E_{z}\right)$ for $F=$ $300 \mathrm{MHz}$.

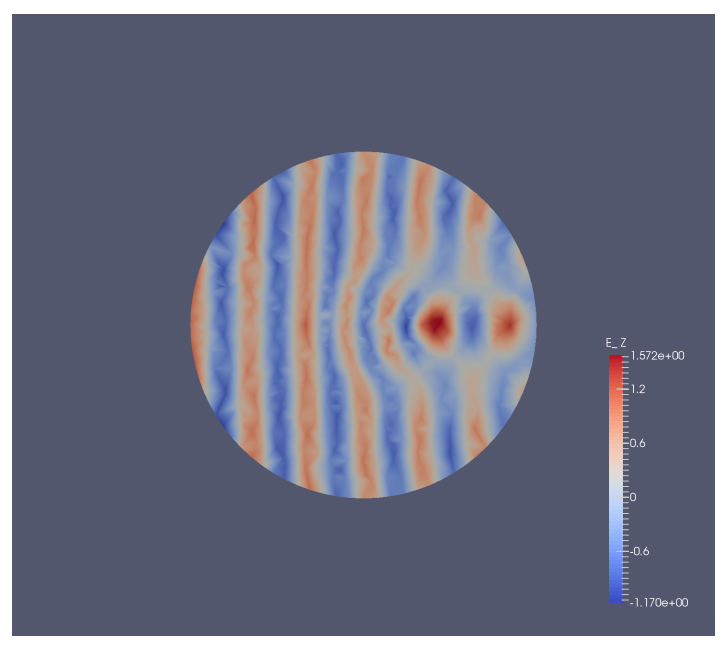

(a) HDG- $\mathbb{P}_{1}$ method.

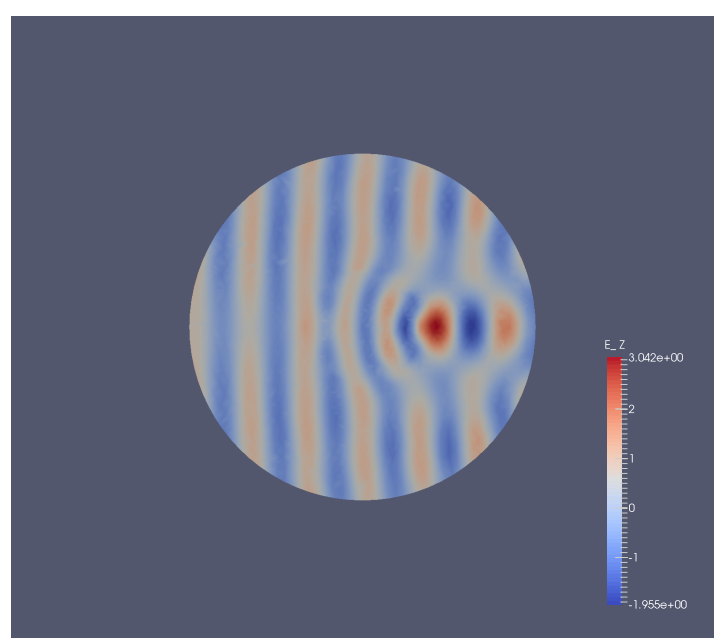

(b) HDG- $\mathbb{P}_{2}$ method.

Figure 4: Scattering of a plane wave by a dielectric sphere: contour lines of $\operatorname{DFT}\left(E_{z}\right)$ for $F=$ $600 \mathrm{MHz}$. 


\begin{tabular}{cccc} 
& \multicolumn{2}{c}{ Implicit } & Explicit \\
& HDG- $\mathbb{P}_{1}$ & HDG- $\mathbb{P}_{2}$ & DG- $\mathbb{P}_{1}$ \\
\hline CFL number & 311 & 311 & 0.82 \\
Matrix order & 547884 & 1095696 & 547848 \\
\# non-zero entries & 22671072 & 90684288 & 3456256 \\
Fill-in ratio (\%) & 0.007 & 0.007 & 0.001 \\
Analysis storage (MB) & 11561 & 43168 & 1664 \\
Factorization storage (MB) & 8863 & 32356 & 1648 \\
Analysis time (s) & 3.0 & 7.4 & 0.4 \\
Factorization time (s) & 174.9 & 1725.4 & 0.236 \\
CPU time (s) & 151 & 11384 & 16362 \\
\hline
\end{tabular}

Table 2: Scattering of a plane wave by a dielectric sphere $F=300 \mathrm{MHz}$ : performance results.

putationally efficient for moderately large 3D problems. In order to address much larger and more realistic problems, a strategy combining explicit and implicit time schemes in the spirit of [23]-[24] will be one of our future works. Beside, improving the accuracy in time is also an important objective. In the context of hybrid explicitimplicit time integration strategies, an original approach inspried from domain decomposition principles has recently been presented in [32] for the second-order wave equation. Whether such an approach can be extended to the first-order form of the system of time-domain Maxwell equations remains an open question that would be worth considering in the HDG discretization framework. Finally, devising a scalable implementation of such an hybrid explicit-implicit HDGTD method will also be a mandatory step.

[1] A. Taflove, S. Hagness, Computational electrodynamics: the finite-difference time-domain method - 3rd ed., Artech House Publishers, 2005.

[2] S. Pernet, X. Ferriéres, G. Cohen, High spatial order finite element method to solve Maxwell's equations in time-domain, IEEE Trans. Ant. and Propag. 53 (9) (2006) 2889-2899.

[3] J. Hesthaven, T. Warburton, Nodal high-order methods on unstructured grids. I. Time-domain solution of Maxwell's equations, J. Comput. Phys. 181 (1) (2002) $186-221$.

[4] V. Kabakian, V. Shankar, W. Hall, Unstructured grid-based discontinuous 
Galerkin method for broadband electromagnetic simulations, J. Sci. Comput. 20 (3) (2004) 405-431.

[5] M. Chen, B. Cockburn, F. Reitich, High-order RKDG methods for computational electromagnetics, J. Sci. Comput. 22-23 (2005) 205-226.

[6] B. Cockburn, F. Li, C.-W. Shu, Locally divergence-free discontinuous Galerkin methods for the Maxwell equations, J. Comp. Phys. 194 (2) (2004) 588-610.

[7] L. Fezoui, S. Lanteri, S. Lohrengel, S. Piperno, Convergence and stability of a discontinuous Galerkin time-domain method for the 3D heterogeneous Maxwell equations on unstructured meshes, ESAIM: Math. Model. Numer. Anal. 39 (6) (2005) 1149-1176.

[8] G. Cohen, X. Ferriéres, S. Pernet, A spatial high order hexahedral discontinuous Galerkin method to solve Maxwell's equations in time-domain, J. Comput. Phys. 217 (2) (2006) 340-363.

[9] S. Dosopoulos, J. Lee, Interconnect and lumped elements modeling in interior penalty discontinuous Galerkin time-domain methods, J. Comput. Phys. 229 (2) (2010) 8521-8536.

[10] S. Dosopoulos, J. Lee, Interior penalty discontinuous Galerkin finite element method for the time-dependent first order Maxwell's equations, IEEE Trans. Ant. and Propag. 58 (12) (2010) 4085-4090.

[11] J. Alvarez, L. Angulo, A. Bretones, S. Garcia, A spurious-free discontinuous Galerkin time-domain method for the accurate modeling of microwave filters, IEEE Trans. Microw. Theory Tech. 60 (8) (2012) 2359-2369.

[12] J. Alvarez, L. Angulo, A. Bretones, S. Garcia, 3-D discontinuous Galerkin timedomain method for anisotropic materials, IEEE Ant. Wir. Prop. Lett. 11 (2012) $1182-1185$

[13] B. Z. S. Dosopoulos, J. F. Lee, Non-conformal and parallel discontinuous Galerkin time domain method for Maxwell's equations: EM analysis of IC packages, J. Comput. Phys. 238 (1) (2013) 48-70.

[14] P. Li, L. Jiang, H. Bagci, Cosimulation of electromagnetics-circuit systems exploiting DGTD and MNA, IEEE Trans. Compon. Pack. Manuf. Tech. 4 (6) (2014) 1052-1061. 
[15] R. Diehl, K. Busch, J. Niegemann, Comparison of low-storage Runge-Kutta schemes for discontinuous Galerkin time-domain simulations of Maxwell's equations, J. Comp. Theor. Nanosc. 7 (2010) 1572.

[16] S. G. A.Hille, R. Kullock, L. M. Eng, Improving nano-optical simulations through curved elements implemented within the discontinuous Galerkin method computational, J. Comp. Theor. Nanosc. 7 (2010) 1581-1586.

[17] M. König, K. Busch, J. Niegemann, The Discontinuous Galerkin Time-Domain method for Maxwell's equations with anisotropic materials, Photonics and Nanostructures - Fundamentals and Applications 8 (4) (2010) 303-309.

[18] K. Busch, M. König, J. Niegemann, Discontinuous Galerkin methods in nanophotonics, Laser and Photonics Reviews 5 (2011) 1-37.

[19] H. Songoro, M. Vogel, Z. Cendes, Keeping time with Maxwell's equations, IEEE Microw. 11 (2) (2010) 42-49.

[20] S. Piperno, Symplectic local time stepping in non-dissipative DGTD methods applied to wave propagation problem, ESAIM: Math. Model. Num. Anal. 40 (5) (2006) 815-841.

[21] M. Grote, T. Mitkova, Explicit local time-stepping methods for Maxwel's equations, J. Comp. Appl. Math. 234 (3283-3302) (2010) 12.

[22] A. Taube, M. Dumbser, C. Munz, R. Schneider, A high-order discontinuous Galerkin method with time-accurate local time stepping for the Maxwell equations, Int. J. Numer. Model. 22 (2009) 77-103.

[23] L. Moya, Temporal convergence of a locally implicit discontinuous Galerkin method for Maxwell's equations, ESAIM Math. Model. Numer. Anal. (M2AN) 46 (2012) 1225-1246.

[24] L. Moya, S. Descombes, S. Lanteri, Locally implicit time integration strategies in a discontinuous Galerkin method for Maxwell's equations, J. Sci. Comp. 56 (1) (2013) 190-218.

[25] B. Cockburn, J. Gopalakrishnan, R. Lazarov, Unified hybridization of discontinuous Galerkin, mixed, and continuous Galerkin methods for second order elliptic problems, SIAM J. Numer. Anal. 47 (2) (2009) 1319-1365. 
[26] N. Nguyen, J. Peraire, Hybridizable discontinuous Galerkin methods for partial differential equations in continuum mechanics, J. Comput. Phys. 231 (18) (2012) $5955-5988$.

[27] N. Nguyen, J. Peraire, B. Cockburn, Hybridizable discontinuous Galerkin methods for the time-harmonic Maxwell's equations, J. Comput. Phys. 230 (19) (2011) 7151-7175.

[28] L. Li, S. Lanteri, R. Perrussel, Numerical investigation of a high order hybridizable discontinuous Galerkin method for 2d time-harmonic Maxwell's equations, COMPEL 2 (3) (2013) 1112-1138.

[29] L. Li, S. Lanteri, R. Perrussel, A hybridizable discontinuous Galerkin method combined to a Schwarz algorithm for the solution of 3d time-harmonic Maxwell's equations, J. Comput. Phys. 256 (2014) 563-581.

[30] E. Hairer, C. Lubich, M. Roche, The numerical solution of differential algebraic systems by Runge-Kutta methods, Vol. 1409 of Lecture Notes in Mathematics, Springer-Verlag, 1989.

[31] P. Amestoy, I. Duff, J.-Y. L'Excellent, Multifrontal parallel distributed symmetric and unsymmetric solvers, Comput. Methods Appl. Mech. Eng. 184 (2000) $501-520$.

[32] J. Chabassier, S. Imperiale, Fourth order energy-preserving locally implicit time discretization for linear wave equations, Int. J. Num. Meth. Engrg. 106 (2015) 593-622. 